\title{
Desktop Analysis Reporting Tool (DART)
}

\section{User's Guide}

\section{Version 4.12.1.1}

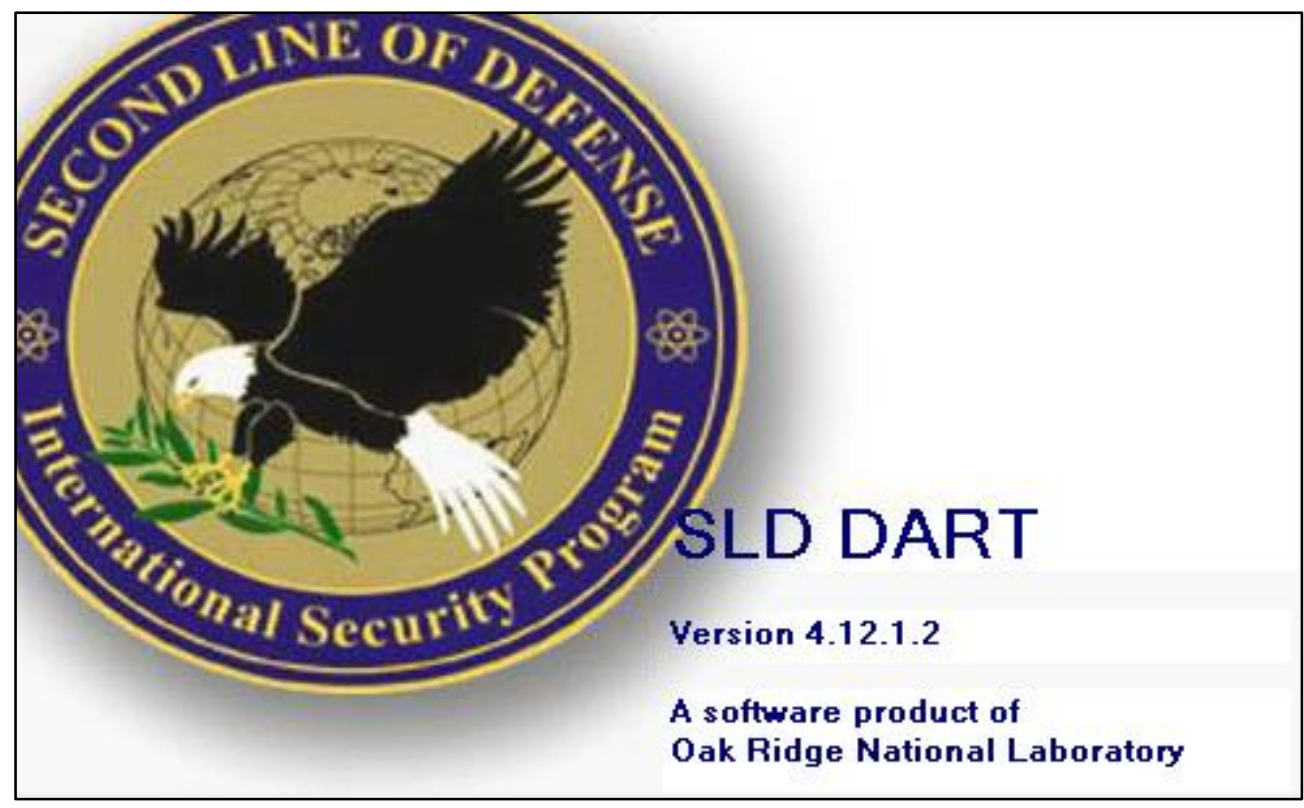

A software product of Oak Ridge National Laboratory

Prepared by: Angela Lousteau

Scott Alcala

March 2012 


\title{
Desktop Analysis Reporting Tool (DART) User's Guide
}

A software product of Oak Ridge National Laboratory

Angela Lousteau

Scott Alcala

\begin{abstract}
This report was prepared as an account of work sponsored by an agency of the United States government. Neither the United States government nor any agency thereof, nor any of their employees, makes any warranty, express or implied, or assumes any legal liability or responsibility for the accuracy, completeness, or usefulness of any information, apparatus, product, or process disclosed, or represents that its use would not infringe privately owned rights. Reference herein to any specific commercial product, process, or service by trade name, trademark, manufacturer, or otherwise, does not necessarily constitute or imply its endorsement, recommendation, or favoring by the United States government or any agency thereof. The views and opinions of authors expressed herein do not necessarily state or reflect those of the United States government or any agency thereof.
\end{abstract}

Date Published: March 2012

Prepared by

Global Nuclear Security Technology Division

Safeguards \& Security Technology Group

OAK RIDGE NATIONAL LABORATORY

Oak Ridge, Tennessee 37831-6283

managed by

UT-BATTELLE, LLC

for the

U.S. DEPARTMENT OF ENERGY

under contract DE-AC05-00OR22725 


\section{Contents}

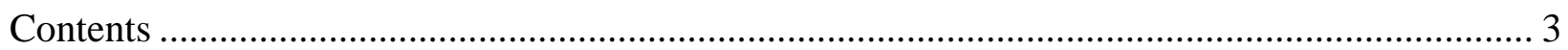

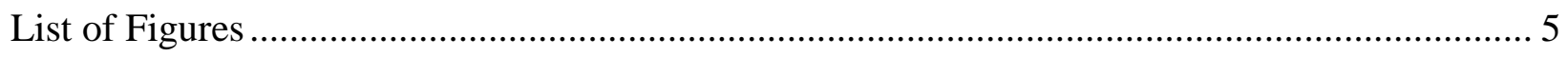

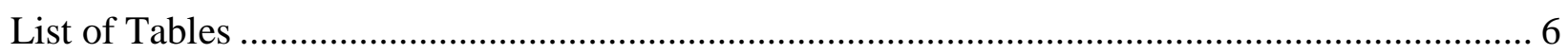

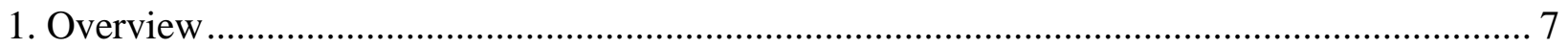

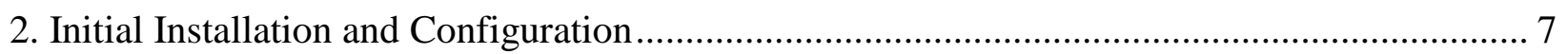

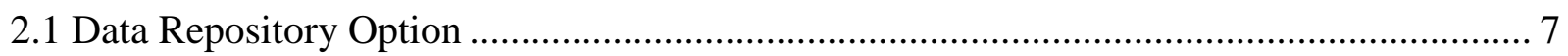

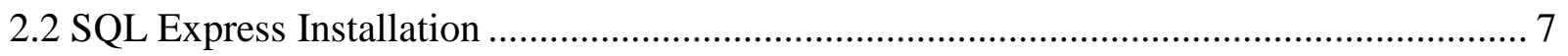

2.3 DART Application Installation............................................................................... 9

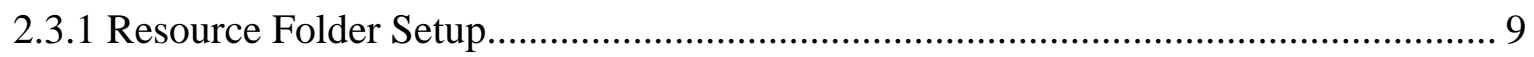

2.3.2 Configuration Settings....................................................................................... 10

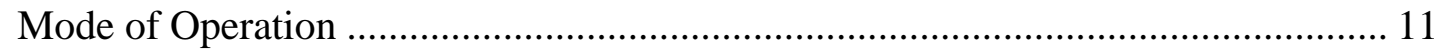

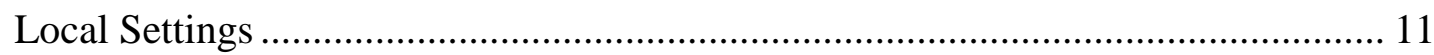

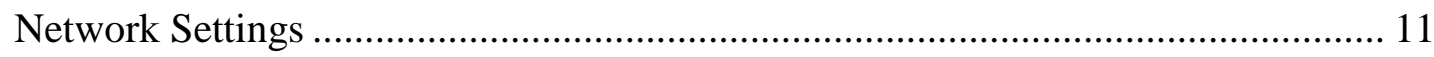

Language Override ……………………......................................................... 12

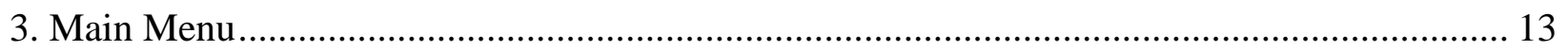

3.1 Administrative Functions........................................................................................ 14

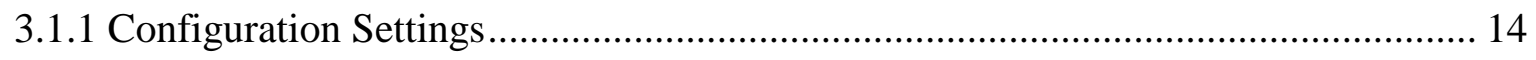

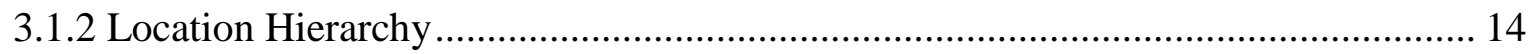

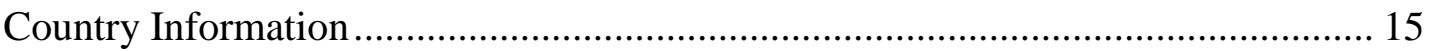

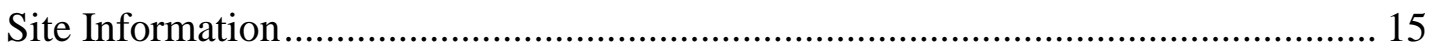

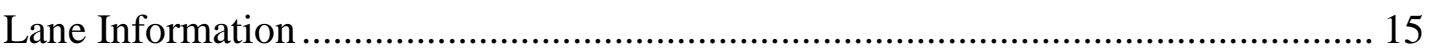

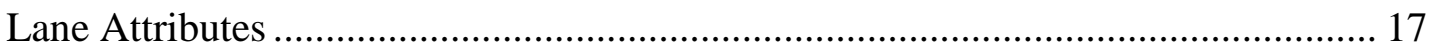

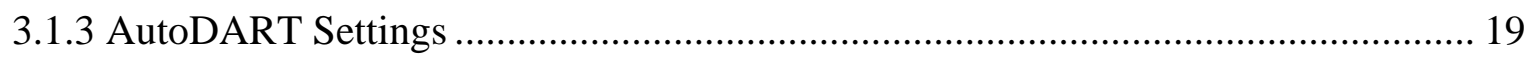

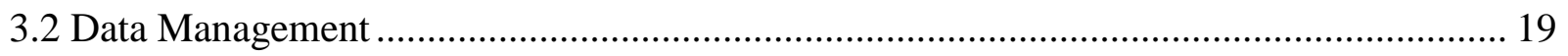

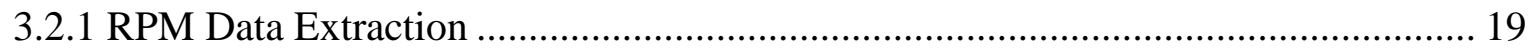

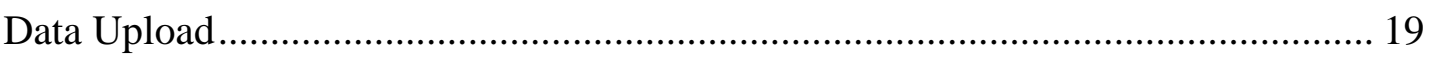

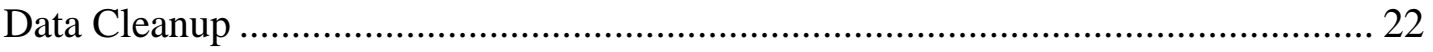

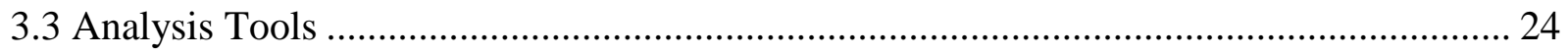

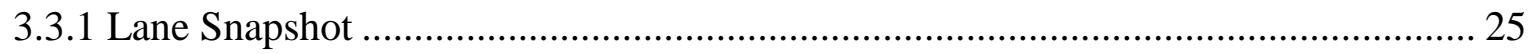

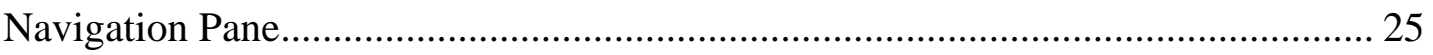

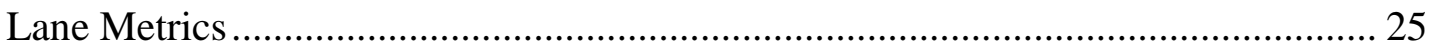

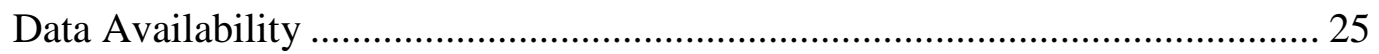

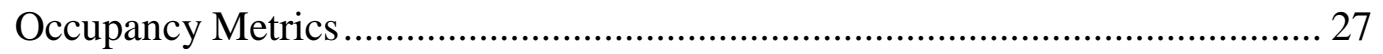

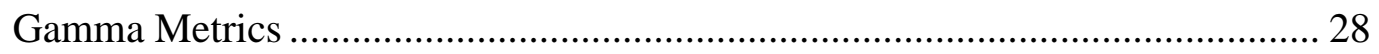

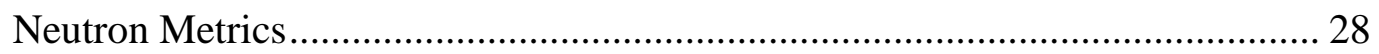




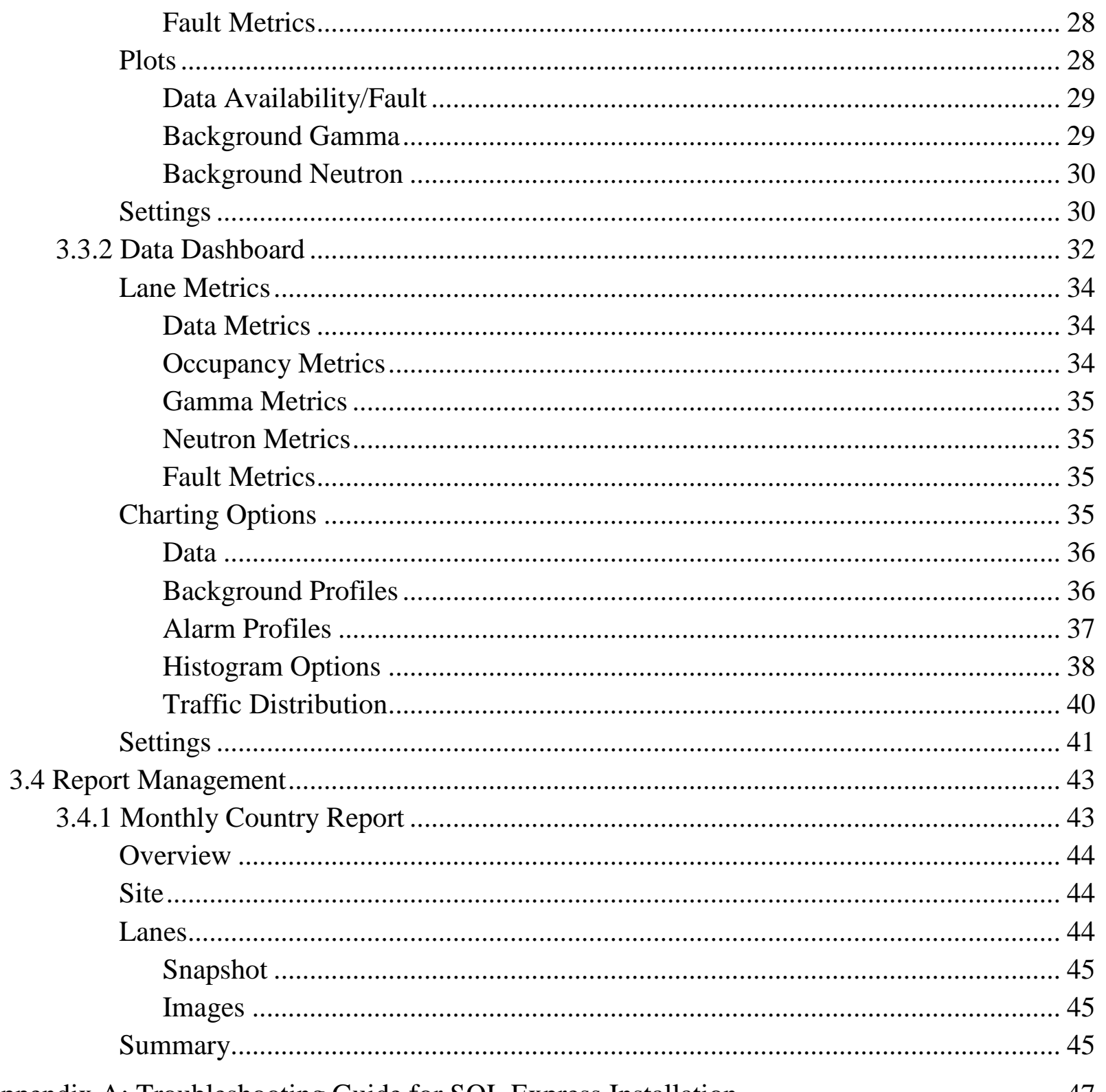

Appendix A: Troubleshooting Guide for SQL Express Installation............................................ 47 


\section{List of Figures}

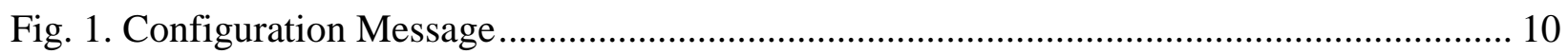

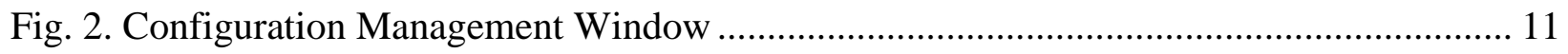

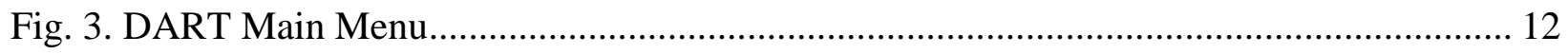

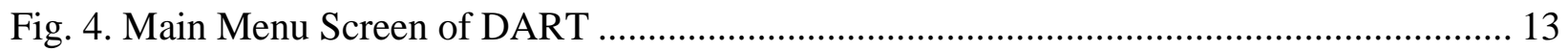

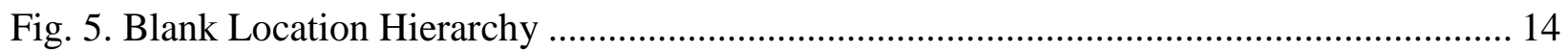

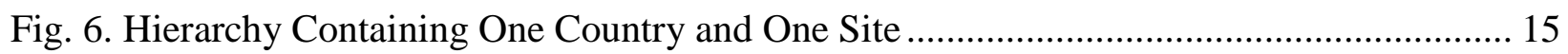

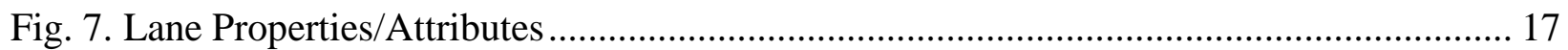

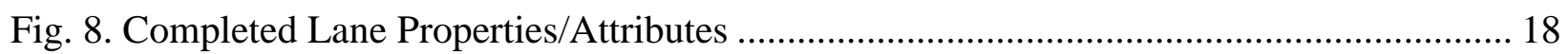

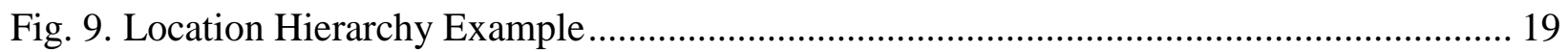

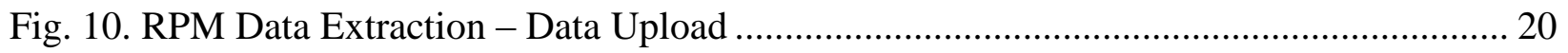

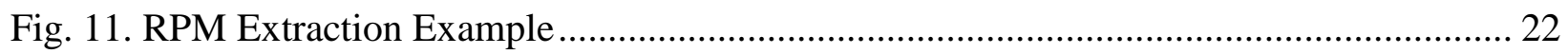

Fig. 12. RPM Data Extraction - Data Cleanup ....................................................................... 23

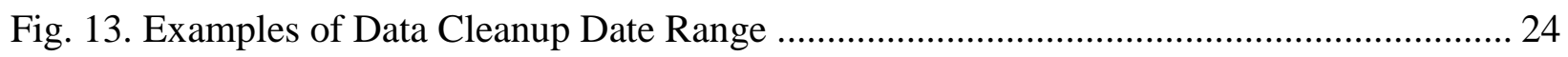

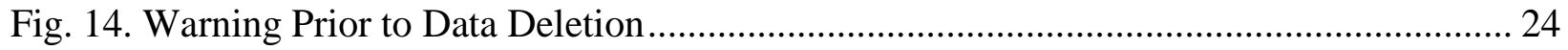

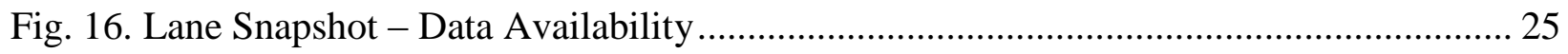

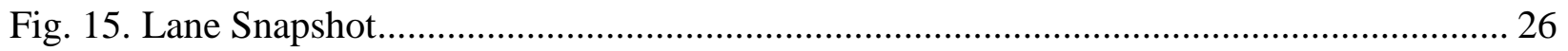

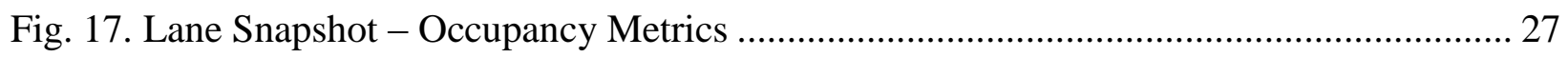

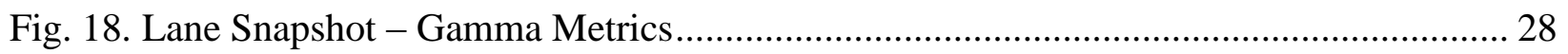

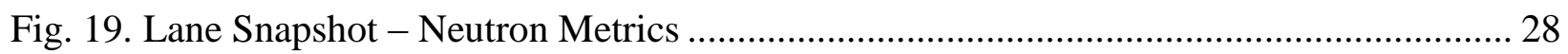

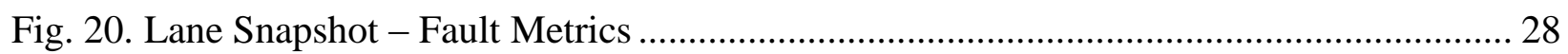

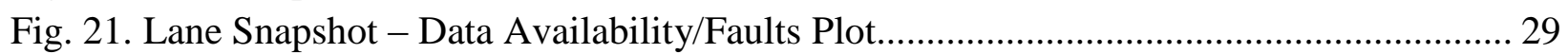

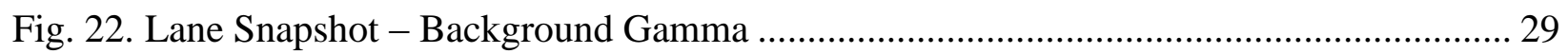

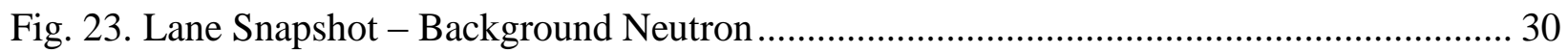

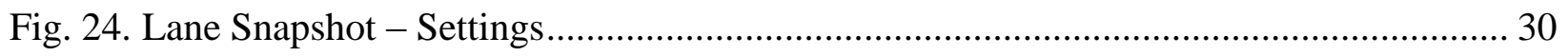

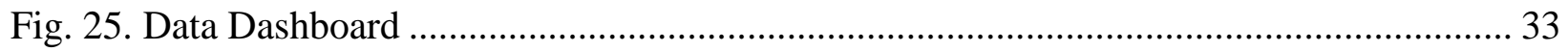

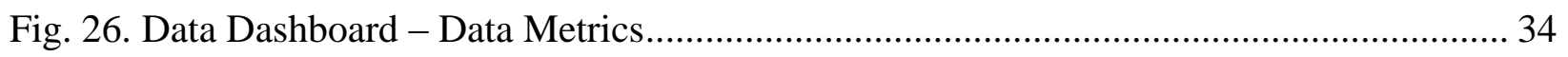

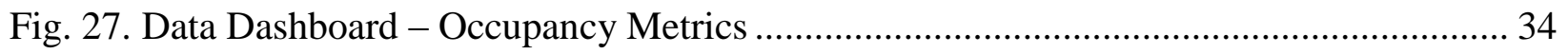

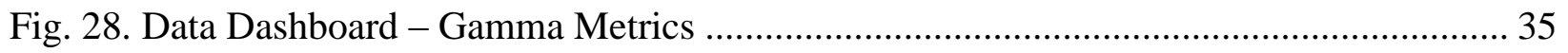

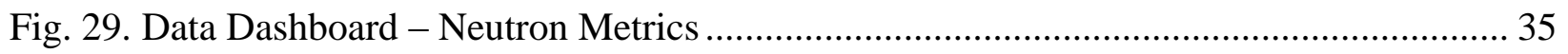

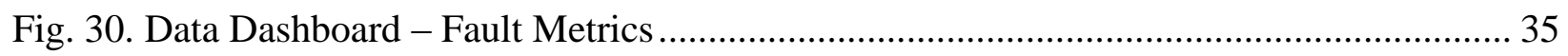

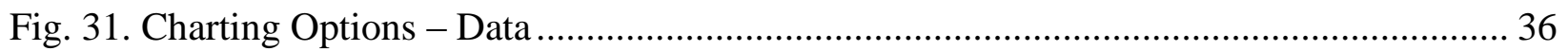

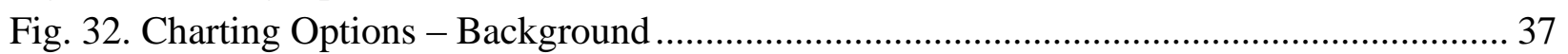

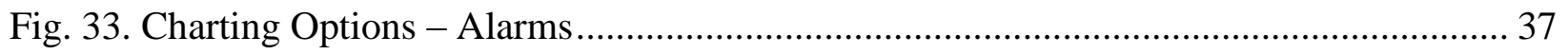

Fig. 34. Charting Options - Histograms - Background Suppression ............................................ 38

Fig. 35. Charting Options - Histograms - Container Length ..................................................... 39

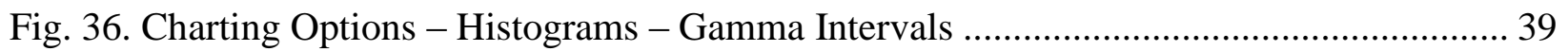

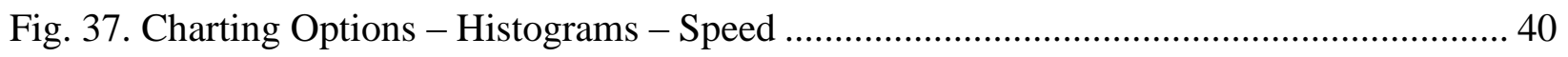

Fig. 38. Charting Options - Histograms ………………...................................................... 40 


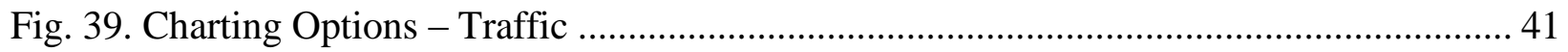

Fig. 40. Data Dashboard - Settings .......................................................................... 41

Fig. 41. Monthly country Report - Country Report List .................................................... 44

Fig. 42. Monthly Country Report - Images ................................................................... 45

Fig. A.1. SQL Server Management Studio ..................................................................... 48

\section{List of Tables}

Table 1. Lane Snapshot - Data Availability Metrics ........................................................... 27

Table 2. Lane Snapshot - Occupancy Metrics................................................................. 27

Table 3. Lane Snapshot - Gamma Settings .................................................................... 31

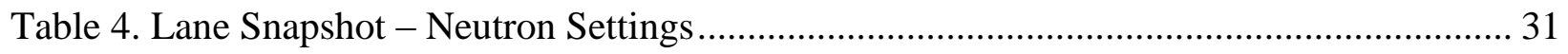

Table 5. Data Dashboard - Occupancy Metrics .................................................................. 34

Table 6. Data Dashboard - Gamma Settings ...................................................................... 42

Table 7. Data Dashboard - Neutron Settings........................................................................ 42

Table 8. Monthly Country Report - Summary Table ....................................................... 46 


\section{Overview}

The Desktop Analysis Reporting Tool (DART) is a software package that allows a user to easily view and analyze radiation portal monitor (RPM) daily files that span long periods. DART gives users the capability to determine the state of health of a monitor, troubleshoot and diagnose problems, and view data in various time frames to perform trend analysis. In short, it converts the data strings written in the daily files into meaningful tables and plots.

DART is an application-based program that was designed to maximize the benefit of a centralized data repository while distributing the workload to individual desktop machines. This networked approach requires a more complex database manager (SQL Server); however, SQL Server is not currently provided with the DART Installation Disk. SQL Express is sufficient for local data analysis and requires the installation of SQL Express and DART on each machine intended for analysis.

\section{Initial Installation and Configuration}

DART utilizes a central repository database for the storage of data extracted from the daily files. DART was designed for use with various data repository configurations. A user's particular configuration will depend on a combination of the work environment and data storage needs.

\subsection{Data Repository Option}

Following are typical data repository and application combinations:

1. SQL Express (personal version of SQL Server) and DART both being installed on a laptop or personal computer. This is the most common repository application. If this is your combination, please proceed to Section 2.2.

2. SQL Server already installed on a network server, and DART being installed on a networked personal computer. If this is your combination, please contact ORNL for detailed instructions before proceeding to Section 2.3.

3. SQL Server and DART both being installed on a laptop or personal computer. If this is your combination, please contact ORNL for detailed instructions before proceeding to Section 2.3.

\subsection{SQL Express Installation}

The current database management program provided with DART is Microsoft SQL Express 2008. Administrator privileges are required for SQL Express installation; however, they are not necessary for use after installation. Once the database manager is installed, the DART Application can be configured. To install SQL Express, follow the subsequent instructions. 
1. Confirm that the computer has Microsoft .NET Framework 2.0 installed by performing the following steps:

a. Control Panel $\rightarrow$ Add/Remove Programs, look for "Microsoft .NET Framework 2.0"

b. If installed, continue with step 2

c. If not installed, it can be installed from the files located in the SQL Install $\rightarrow \mathrm{XP}$ Patches folder on your DART Installation Disk. First, run the executable in the "dotNetfx35" folder. Afterwards, run the executable in the "afterDotNetfx35" folder in the following order:

i. WindowsXP-KB942288-v3-x86.exe

ii. NDP30SP2-KB958483-x86.exe

iii. NDP35SP1-KB958484-x86.exe

These files can also be downloaded and installed from the Microsoft website: http://www.microsoft.com/downloads/details.aspx?FamilyID=0856EACB-4362-4B0D-8EDD$\underline{\mathrm{AAB} 15 \mathrm{C} 5 \mathrm{E} 04 \mathrm{~F} 5}$

Once complete, continue with step 2.

2. Check your computer for old or existing versions of Microsoft SQL Express 2008 by performing the following steps:

a. Control Panel $\rightarrow$ Settings $\rightarrow$ Add / Remove Programs, look for "Microsoft SQL Server 2008"

b. If the latest version of SQL Express is installed, continue with the DART Application Installation. Note that the total storage capacity for SQL Express is 10 GB.

c. If not installed, install SQL Express by running the appropriate executable in the "SQL Install" folder (SQLEXPR32_x86_ENU.exe for 32bit machines or SQLEXPR_x64_ENU.exe for 64bit machines). These files can also be downloaded and installed from the Microsoft website:

http://www.microsoft.com/express/Database/

d. Accept the End User License Agreement and take all defaults during installation. Once a successful installation is complete, proceed to the DART Application Installation section

For troubleshooting tips on SQL Express installation, see Appendix A. 


\subsection{DART Application Installation}

The following steps will complete DART installation.

1. Copy the "DART Application" folder to the root directory of the computer being used for analysis. This will typically be c:IDART Application. Some of the files contained in this folder will be used only during the setup process, but this is a convenient place to keep them for future reference. The files in red type are used each time DART is accessed.

a. ceTe.DynamicPDF.20.dll

b. DART.bak - for SQL Server

c. dart.exe-DART application

d. DART Commodity Classification.pdf

e. DART Db Creation.txt - for use with SQLServer

f. DART User Guide.doc

g. dartData.mdf - the database in which your extracted data will be stored

h. DundasWinChart.dll

i. en folder - language support (.dll file must be located in this subfolder)

j. es folder - language support (.dll file must be located in this subfolder)

2. Create a shortcut to "dart.exe" on your desktop workspace.

Note: when opening the DART Application directory any time after the initial use, the above list will also include "config.xml". This file contains settings specific to your installation (created in a later step). If the local database option (SQL Express) is being used, the list will also include a "dartData_log.ldf" file after the first connection has been established.

\subsubsection{Resource Folder Setup}

DART utilizes a number of directory folders to manage and organize daily files, images, and reports. These folders are created the first time that DART is accessed, but their locations will need to be specified:

- If DART will be used from a single desktop or laptop computer, the resource folder can be created on that machine 
- If DART will be used by multiple people in a networked environment, the resource folders should be created in a location accessible to everyone. This can be the same physical machine on which SQL Server is installed, or some other networked computer

Regardless of the location, users must have sufficient permission to create new subfolders, as well as create, move, and delete files within this folder structure.

Copy the "DART Files" folder from the DART Installation Disk to the desired location. This will typically be the root directory c:, resulting in the following resource folder path: c:IDART Files. Remember this location; you will need to enter this specific location into the configuration step later.

\subsubsection{Configuration Settings}

The following items must be completed before DART can be accessed for normal daily use. After the initial setup, some of these values will never change. Others will change only infrequently and can be accessed as needed.

Double click the DART shortcut icon on the desktop. A "splash screen" will appear, followed almost immediately by a message box stating that the configuration file could not be located. This standard message (Fig. 1) is displayed when connecting to DART for the first time.

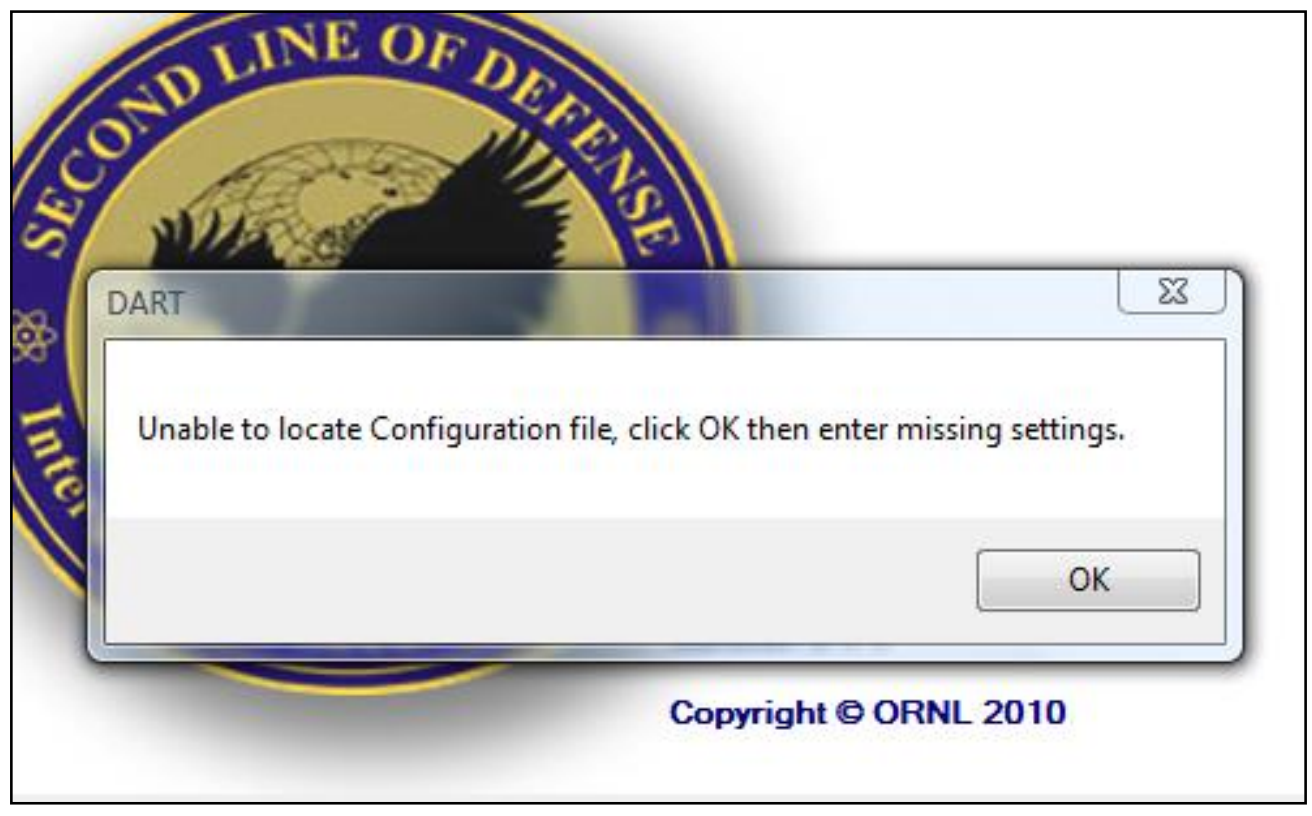

Fig. 1. Configuration Message

After clicking $O k$, you will see the Configuration Management window (Fig. 2). 


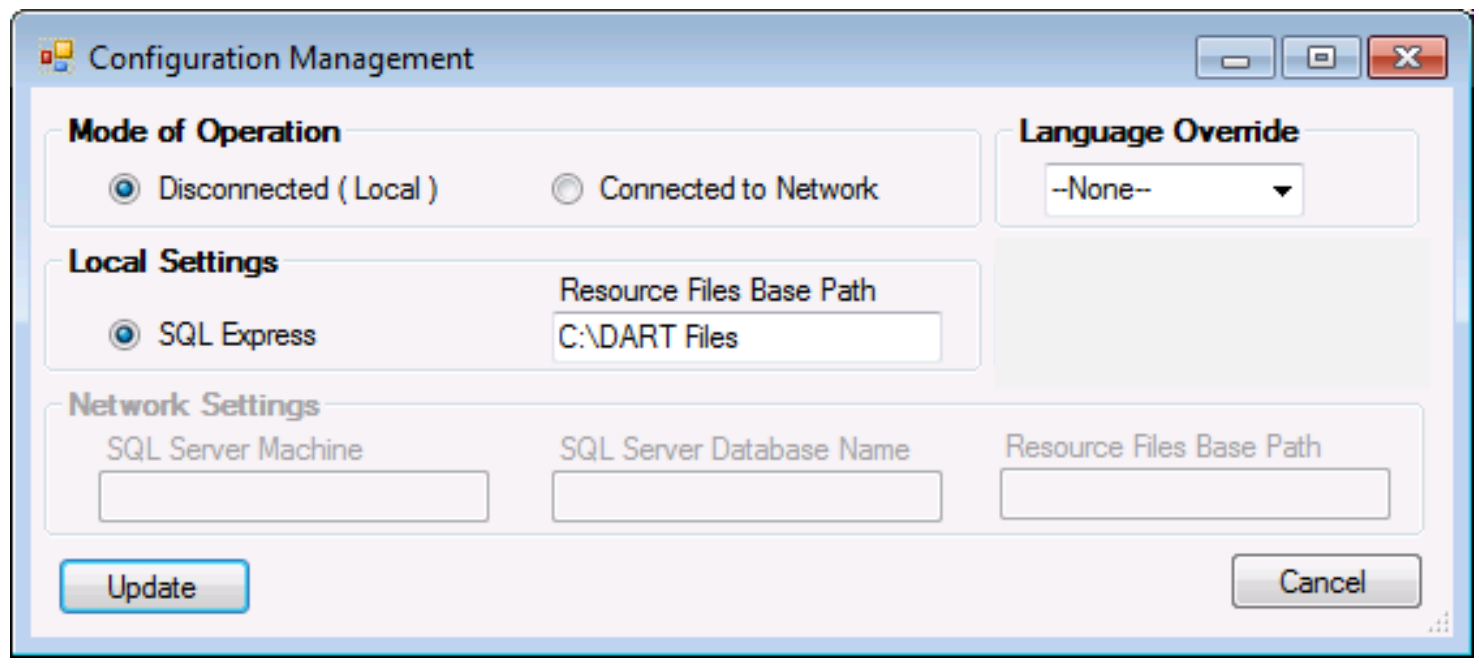

Fig. 2. Configuration Management Window

The window is divided into Mode of Operation, Local Settings, Network Settings, and Language Override.

\section{Mode of Operation}

There are two options for Mode of Operation: Disconnected (Local) and Connected to Network. If SQL Express is being used, you should operate in the Disconnected (Local) Mode. By selecting Disconnected, the Local Settings section will be available for entry. If SQL Server is being used and the data will be accessed through a network, the Network Settings section will be available for entry.

\section{Local Settings}

1. Select SQL Express (MySQL is not currently supported)

2. Resource Files Base Path: The path and folder created in the Resource Folder Setup section of this guide. If this is a networked location, the value entered should look something like IIshareNamelDART Files. If it is a local location, it will typically be c:IDART Files

\section{Network Settings}

1. SQL Server Machine: This is the URL or shared name of the machine on which SQL Server is installed, as instructed in Appendix B: SQL Server Database Creation

2. SQL Server Database Name: All installations outside Oak Ridge National Laboratory should use "DART" for this value

3. Resource Files Base Path: The path and folder created in the Resource Folder Setup section of this guide. If this is a networked location, it should look something like IIshareNamelDART Files. If it is a local location, it will typically be c:IDART Files 


\section{Language Override}

DART provides multi-language support for specific language options. By default the language specified by your computer configuration will be displayed, providing DART has a dictionary for that language. To select a different language, click the drop-down menu under Language Override and choose from the available options. After clicking Update, the menu should be redisplayed in the language selected.

Once the appropriate values are entered, click Update. DART will try to connect to the database at this point. If errors are encountered during this process, a message will be displayed. If the connection and folder creation were successful, the configuration information will be written to the "config.xml" file and saved within the "DART Application" folder. If you receive database connection errors, please see Appendix A and/or B for additional help.

After the initial configuration has been completed successfully, the DART Main Menu will be displayed. If the connection is successful, the main menu will contain several links in blue type (Fig. 3). If connection to the data repository was not successful, the menu options will be grayed out and only the Close Application button will function. This will only be true if you have clicked Cancel from the Configuration Management window.

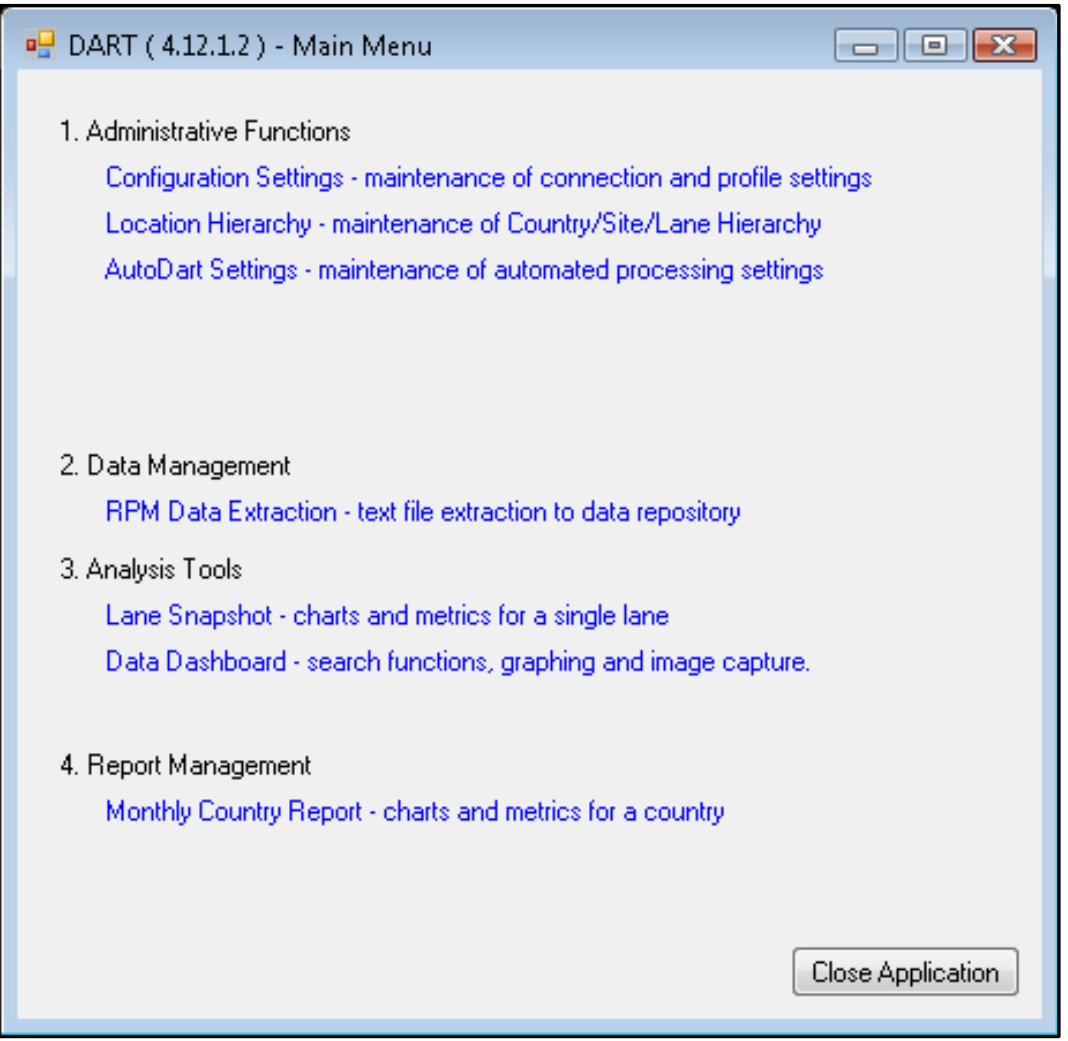

Fig. 3. DART Main Menu 


\section{Main Menu}

Upon successful completion of the configuration settings, the user will be directed to the DART Main Menu (Fig. 4). Note: As long as the configuration settings remain valid, the user will automatically be directed to the DART Main Menu, not the Configuration Settings Menu, upon connection.

DART is opened for normal use by double-clicking the DART shortcut on the desktop workspace. After an initial "splash screen," the Main Menu is presented. The Main Menu is divided into four groups of functionality, each with one or more options. These options appear as active blue text if the user has successfully

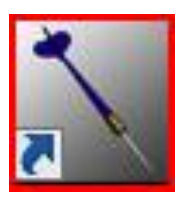
connected to the SQL database; otherwise, they are gray.

Click the blue text to proceed with the associated option. These options are discussed in detail in the following sections.

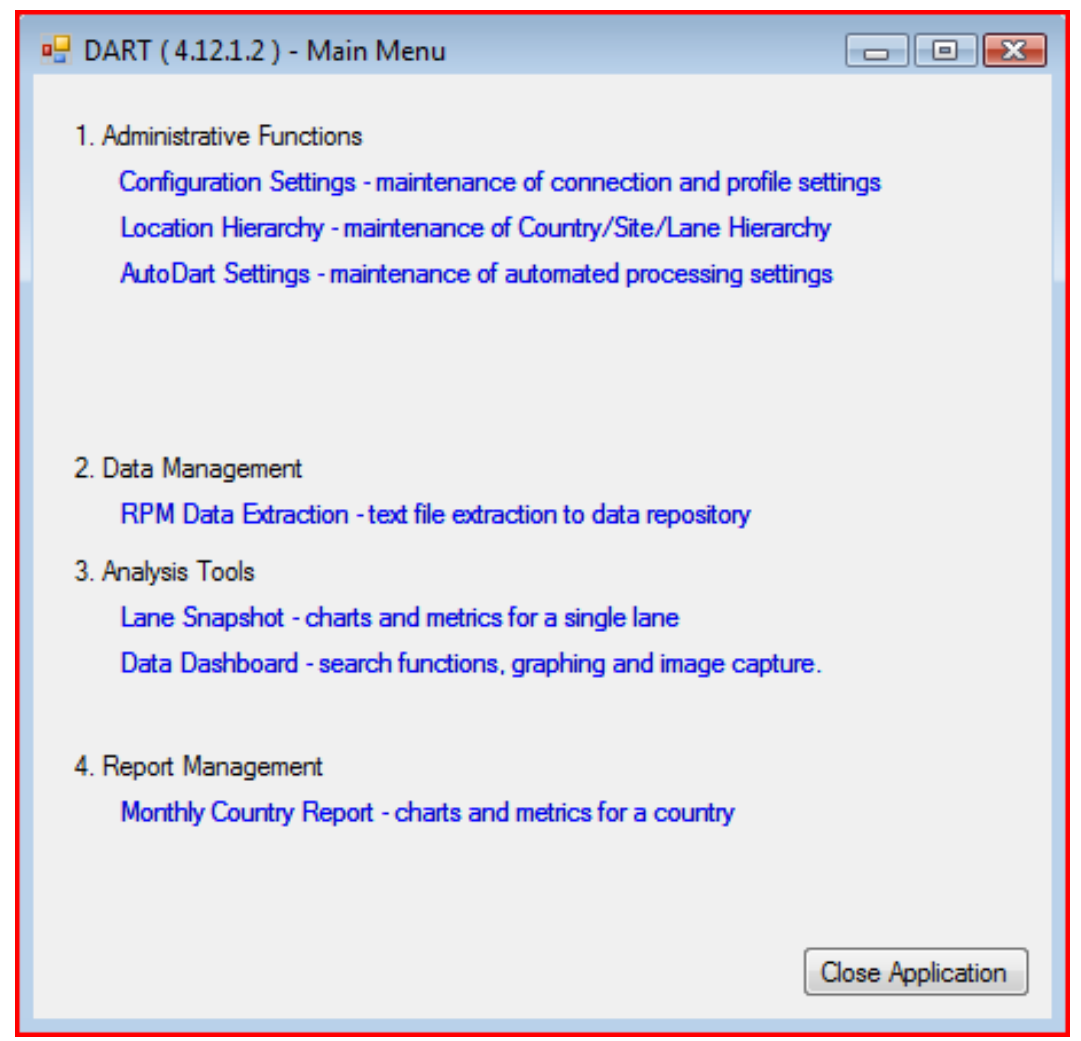

Fig. 4. Main Menu Screen of DART

The Close Application button ends the DART application session. 


\subsection{Administrative Functions}

Administrative Functions grant the user access to settings and values that control the database connectivity and determine how DART links the data from the daily files to individual lanes.

\subsubsection{Configuration Settings}

By clicking Configuration Settings in the main menu, the user has access to the same configuration management parameters that were set up during the initial DART configuration (see Section 2.3.2). These settings do not typically change after the initial setup; however, should the users need to change them, they are accessible.

\subsubsection{Location Hierarchy}

Setting up the Location Hierarchy is an integral step in DART. The Location Hierarchy not only defines the relationships between countries, sites, and lanes, it also provides the required association between a lane and the corresponding daily files through the Upload File Pattern. This Upload File Pattern tells DART which daily files belong to which lanes. It is vital that this information be set up correctly prior to any data extraction (discussed in Section 3.2.1).

Figure 5 shows the Location Hierarchy as it appears the first time it is accessed. The left window is called the Navigation Pane and allows the user to switch between elements (in this case, elements will be countries, sites, and/or lanes). The right window contains specific values associated with various components of the location hierarchy.

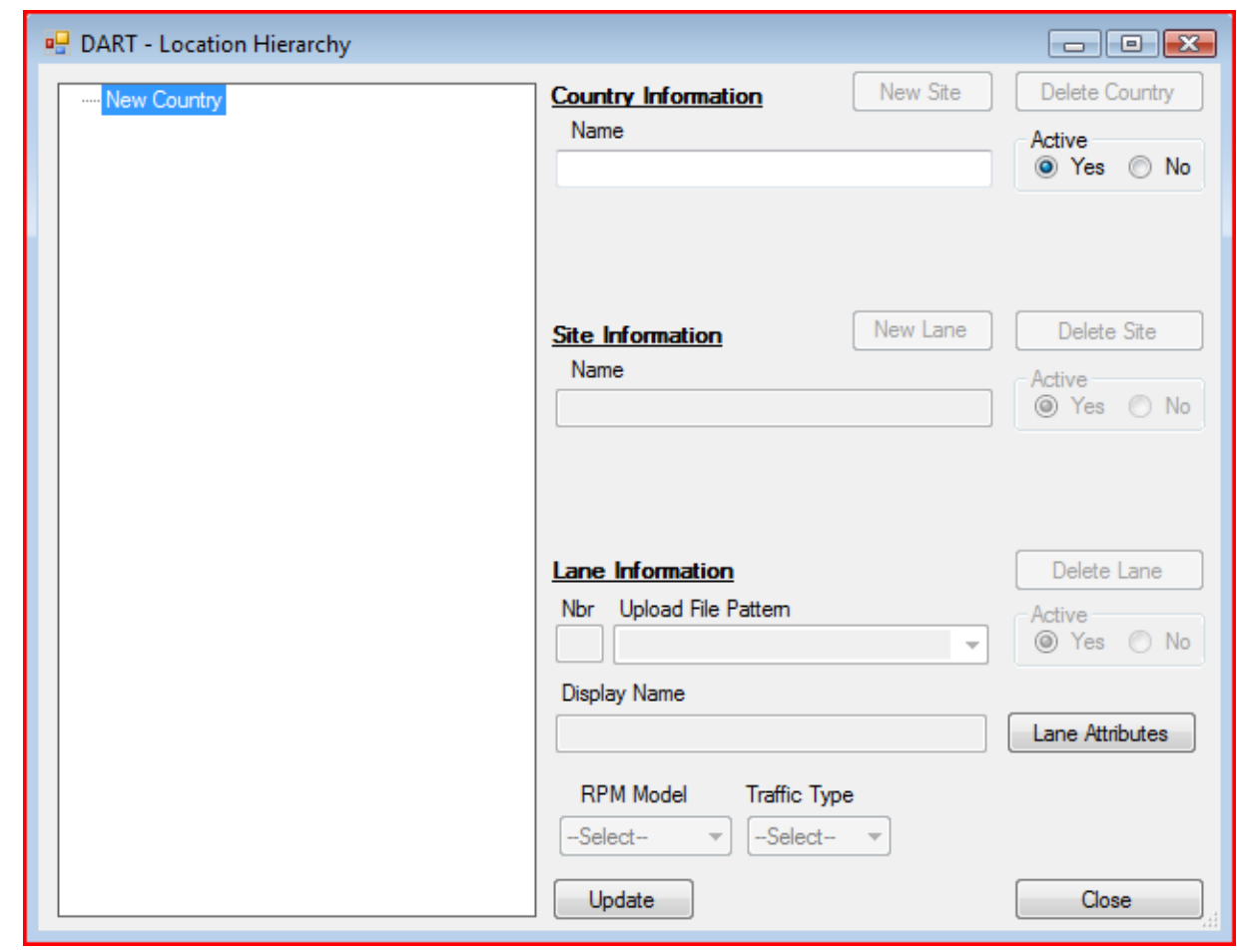

Fig. 5. Blank Location Hierarchy 


\section{Country Information}

Setup of the Location Hierarchy must begin with the addition of a new country. First, enter the country name in the Name field. By default, the status in the Active box will be Yes. Click the Update button to accept the new country. The page should refresh and the country should appear in the Navigation Pane on the left. This can be repeated for multiple countries.

\section{Site Information}

Sites may now be added to this country; a country may have more than one site. To add a site, click the country name in the Navigation Pane. Then click the New Site button next to Country Information on the right, which should now be enabled. Under Site Information, the site name can then be added to the Name field. Again, the status in the Active box should be YES. Click the Update button to accept the new site. The page should refresh and the country in the Navigation Pane should now have a $[+]$ sign, indicating subordinate elements (sites are subordinate to countries) that can be expanded or collapsed for readability.

At this point, another site can be added by clicking the New Site button while the country is highlighted, or lanes can be added to the newly added site. Figure 6 shows the Navigation Pane with the addition of a country (called My Country) and its site (called My Site).

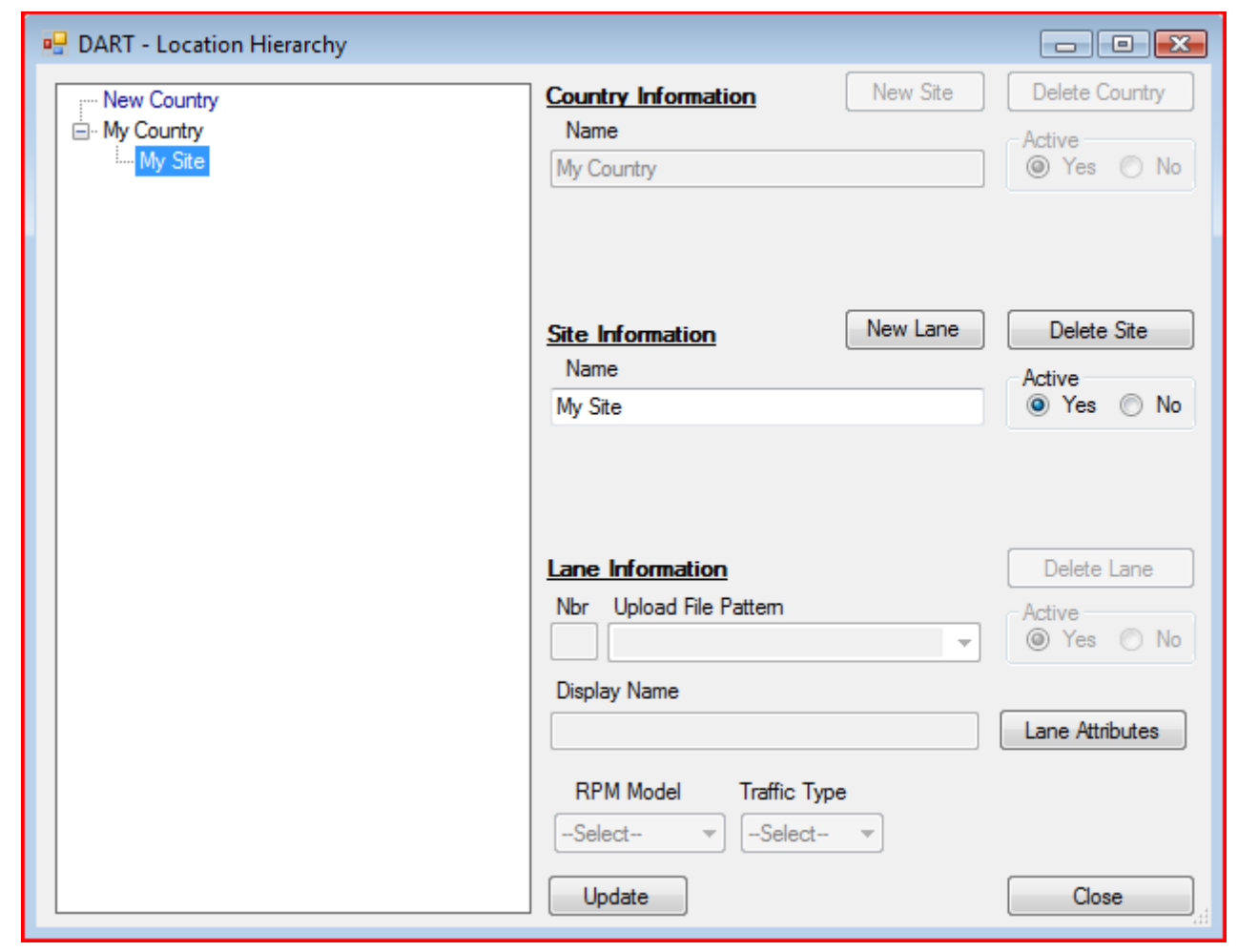

Fig. 6. Hierarchy Containing One Country and One Site

\section{Lane Information}

To add a new lane, first highlight in the Navigation Pane the site to which the lane will belong. In this case, My Site is highlighted. Then click the New Lane button. This will enable the Lane 
Information section on the right. First, the user should enter a number in the Nbr field. This number is used as the primary sort column for the list of lanes within DART. If your site uses lane numbers as part of the naming convention, that number *should* be used in the Nbr field to reduce confusion. Otherwise, number the lanes in the order in which you want them to appear in DART. Lane numbers need not be sequential, but they must be unique.

The Upload File Pattern allows DART to associate a group of daily files with a particular lane. As such, it is vital that this value be entered correctly prior to performing data extractions for that lane. To enter this value correctly, the name of the daily file for each lane must be known. Having the daily files ahead of time will expedite the setup of the Upload File Pattern.

The published convention for daily file names dictates that the file name be composed of six

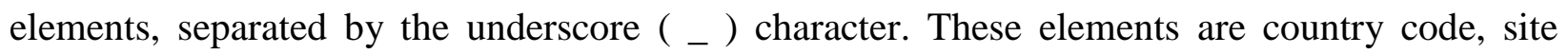
name, lane number, equipment type, software version, and date. A file named in accordance with this convention would look like the following:

MC_MySite_L001_Tvm250_v001_2010-01-25.txt

For the Upload File Pattern, we are concerned only with enough of the name to determine Country, Site, and Lane. For our example, this would be the following:

\section{MC_MySite_L001}

This is the value that should appear in the Upload File Pattern. It does not contain equipment type, software version, or date information.

If the daily files are readily available, copy them directly to the "Data" resource folder. This was set up earlier in the Resource Folder Setup section and will typically be c:IDART Files $\backslash$ Data. If the daily files are located in this folder, they will appear in the drop-down menu in the right corner of the Upload File Pattern field on the right side of the Location Hierarchy screen. This helps ensure that no mistakes or typos are made when entering this value. If the daily files are not readily available, the Upload File Pattern can be typed into the field.

Again, the Active field should be left as Yes. You may enter a Display Name in the field beneath

the Upload File Pattern. This display name can be anything used to identify the lane. For example, the daily file name may call the lane L001, but it may be referred to as Export Lane 1 at the site. Export Lane 1 can be used as the Display Name so that the lane is easily identifiable.

At this point, clicking Update will add a lane. The page should refresh and the site in the Navigation Pane should now have a [+] sign (lanes are subordinate to sites). Note: A lane cannot exist without a site, and a site cannot exist without a country. 


\section{Lane Attributes}

Additional properties can be added to each lane by clicking the Lane Attribute button in the Lane Information section of the Location Hierarchy. The Lane Properties window will appear as shown in Fig. 7. This window allows the user to assign a Monitor Type, Traffic Type, and Portal Monitor to each lane.

Portal Monitor refers to the model of RPM, for example, SLD1P, SLDVH, or SLDT. This is especially helpful for quickly distinguishing single-pillar pedestrian monitors from dual-pillar pedestrian monitors during DART analysis. Monitor Type is a useful way to classify portal monitor type (e.g., vehicle monitor). Traffic Type refers to the way in which the RPM is being used (e.g., rail monitors being used to monitor vehicle traffic instead of railcars). This is helpful when looking at things like speeds, background suppression, and cargo distributions (see Section 3.3.2).

When starting with a new installation of DART, there will be no Monitor Types, Traffic Types, or Portal Types listed.

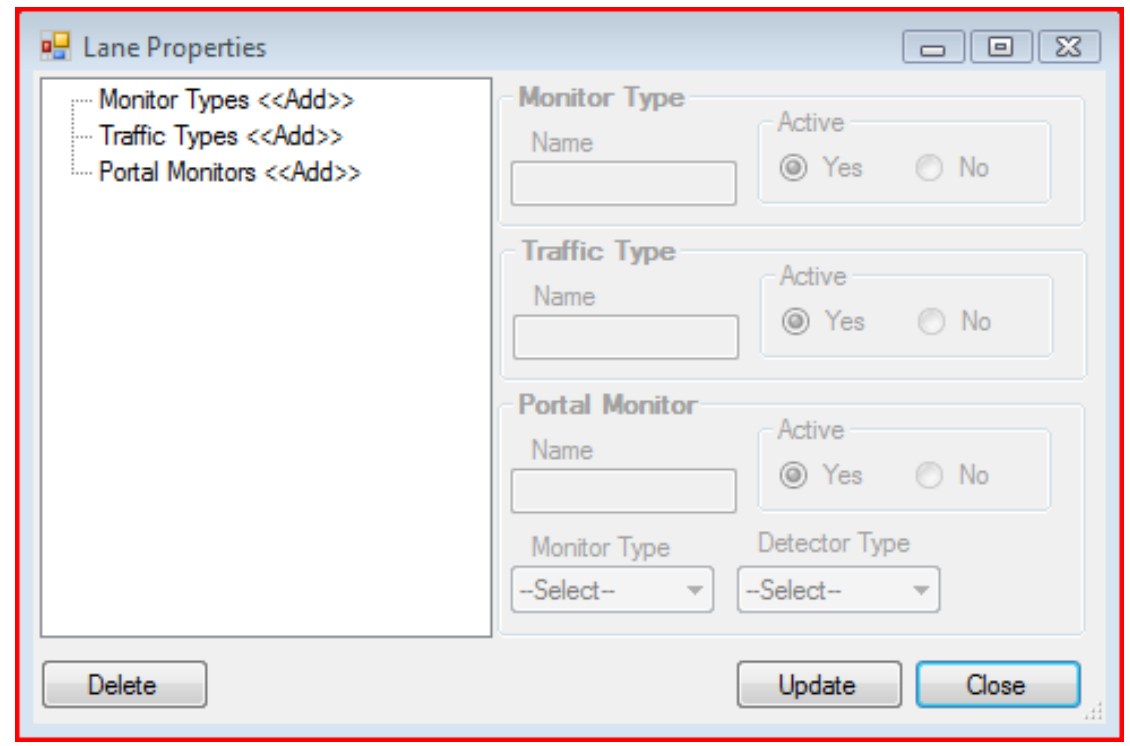

Fig. 7. Lane Properties/Attributes

To begin, highlight Monitor Types in the Navigation Pane on the left. Enter a Monitor Type relevant to the country of interest in the Name field (i.e., Conveyor, Pedestrian, Vehicle, or Rail). Click the Update button. The Navigation Pane on the left will refresh and there will be a [+] sign next to Monitor Types indicating the addition. Continue adding Monitor Types as needed.

Add traffic types by selecting Traffic Types in the Navigation Pane on the left. Enter the Traffic Type in the Name field in the Traffic Type section on the right (i.e., Conveyor, Pedestrian, Vehicle, or Rail). Click the Update button to refresh the Navigation Pane. Continue adding Traffic Types as needed. 
To enter the Portal Monitors attribute, click and highlight Portal Monitors in the Navigation Pane. In the Name field, enter the hardware type (e.g., SLD1P, SLDVH, or SLDT). From the Monitor Type drop-down menu under the Portal Monitor section, choose the Monitor Type that corresponds to this lane and Portal Monitor. From the Detector Type drop-down menu, choose RPM. Note: this version of DART does not support Spectroscopic Portal Monitor (SPM) daily files. Click the Update button to refresh the Navigation Pane. Continue adding Portal Monitors as needed. Once complete, the Lane Properties should look similar to Fig. 8.

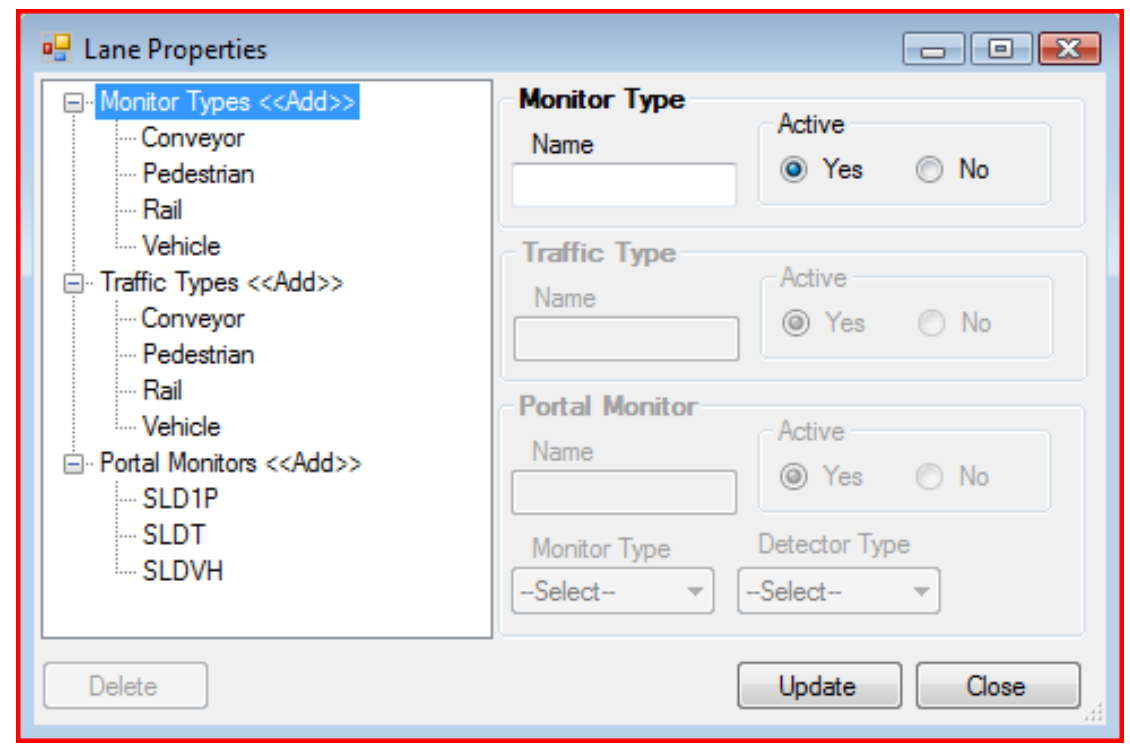

Fig. 8. Completed Lane Properties/Attributes

When all the Lane Properties have been entered and updated, click the Close button. This will return the user to the Location Hierarchy screen. By clicking on a lane in the Navigation Pane, an RPM Model (based on Portal Monitors added in Lane Attributes) can be specified as well as a Traffic Type (based on options added in Lane Attributes). Note that lanes without attribute information will not be displayed in the MCR Navigation Pane (see Section 3.4.1).

Figure 9 shows a location hierarchy for a country (named My Country) with two sites (My Site and Your Site). Each site has two lanes.

Once all sites and lanes are added and updated, click the Close button to return to the DART Main Menu. 


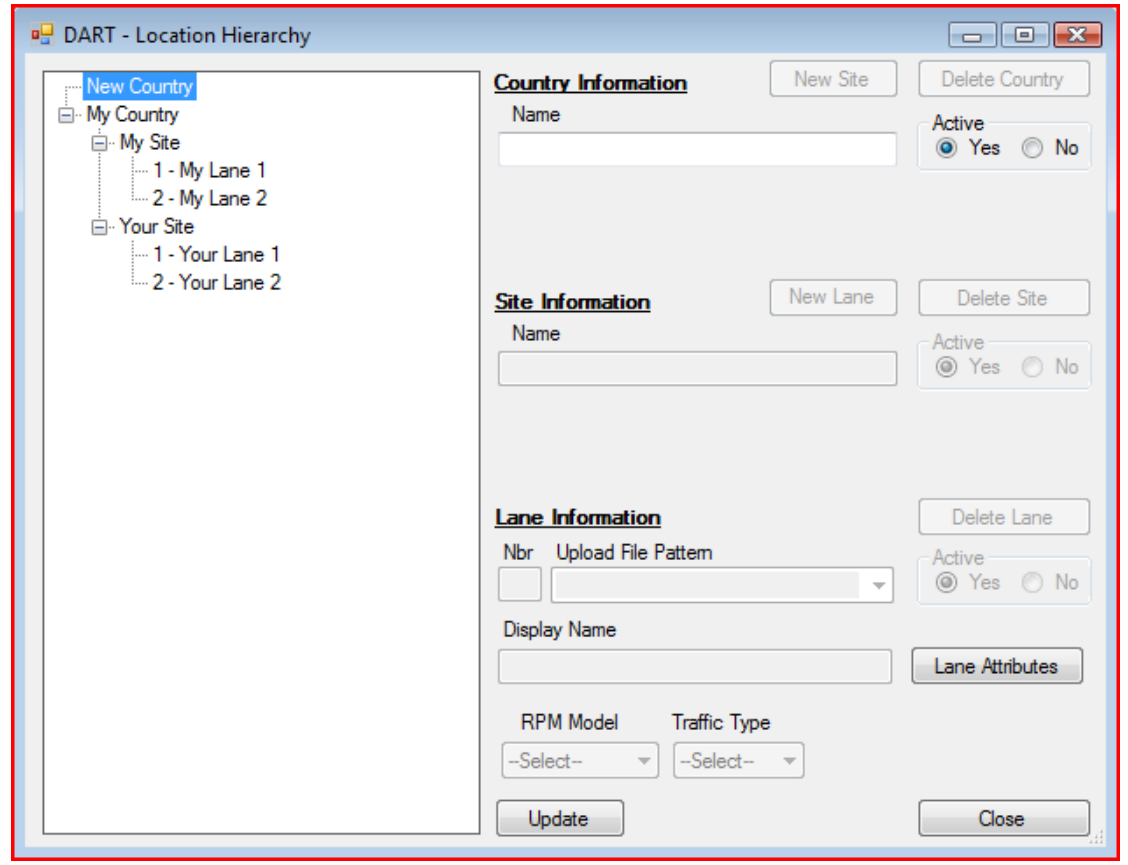

Fig. 9. Location Hierarchy Example

\subsubsection{AutoDART Settings}

AutoDART is a companion module (separate executable) that allows DART to automatically process daily files on a scheduled basis. It can be configured to process daily files, remove old data from the SQL database, and automatically generate reports. Use of this module requires additional levels of configuration and assistance from ORNL. Please contact ORNL to employ this option.

\subsection{Data Management}

The role of Data Management is to process the daily files. In the processing of daily files, also referred to as data extraction, DART reads the daily files and extracts or transfers important data to the SQL database for analysis and reporting.

\subsubsection{RPM Data Extraction}

RPM Data Extraction provides the mechanism for processing daily files into an SQL database that can be easily used by the analysis and reporting features (discussed later). In addition to processing new daily files, the RPM Extraction Tool allows users to easily eliminate old data from the SQL database. Only daily files in the "Data" resource folder will be processed (typically c: $\mid D A R T$ Files $\backslash$ Data).

\section{Data Upload}

The Data Upload tab at the top of the RPM Data Extraction screen is divided into three panes: the Navigation Pane on the left, which displays countries and sites; the Site Metrics Pane in the upper right corner, which is used to display site-specific metrics; and the Results Pane in the 
lower right corner, which uses various messages to communicate the results of the data extraction process (Fig. 10). To display the site metrics for a given site, click on the site in the Navigation Pane to the left. If no daily files have been processed, N/A and [0] values will appear in each element of the Site Metrics Pane.

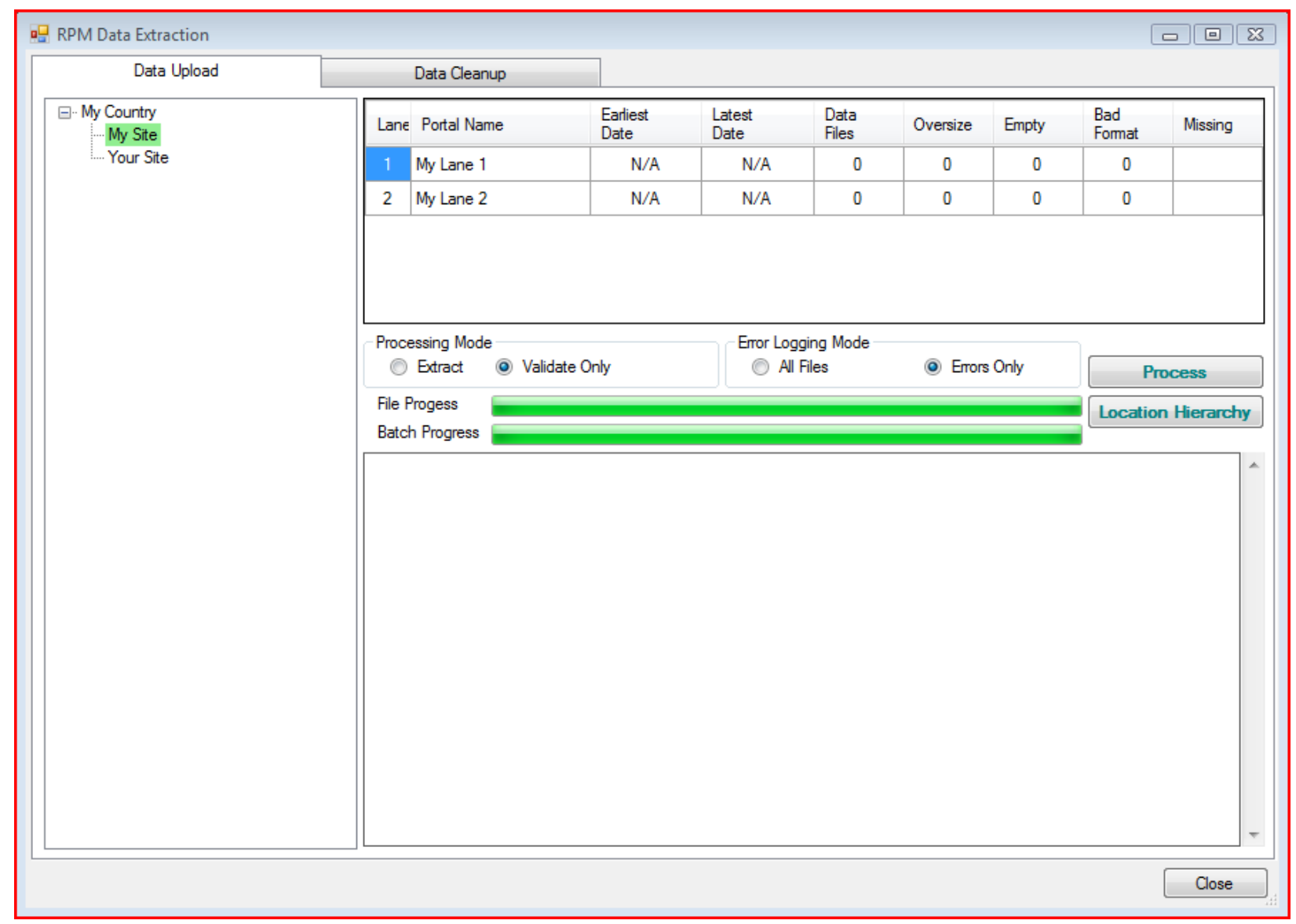

Fig. 10. RPM Data Extraction - Data Upload

In the middle of the Data Upload tab are two groups of radio buttons, two progress bars, and two action buttons (Process and Location Hierarchy). The Processing Mode section has two radio button options: Extract and Validate Only. This setting will determine what happens when the Process button is clicked. The Validate Only option will check that the daily files waiting to be processed (in the c:IDART Files\Data folder) are associated with a lane in the Location Hierarchy via the Upload File Pattern; DART knows to which lane they belong. If a daily file cannot be validated, the Upload File Pattern is likely set up incorrectly in the Location Hierarchy. To navigate directly to the Location Hierarchy, simply click the Location Hierarchy button.

No data extraction will occur when Validate Only is selected. If, however, the Extract option is selected when the Process button is clicked, DART will perform the validation and then proceed with data extraction for the files that were validated. For each of these actions, the progress bars 
will reflect the percentage of completion for the current file being processed (File Progress) and the percentage of completion for the entire group of files being processed (Batch Progress). The progress bars will advance as long as the RPM Data Extraction page has focus; if you click off the window to initiate some of other computer activity, the bars will stop advancing even though the application is still running and the data is still being extracted. When the extraction process is complete, the Results Pane will update according to the Error Logging Mode option selected.

Depending on the Error Logging Mode selection, messages will appear in the Results Pane upon completion of the task. If the All Files option is selected, a message will appear for each file in the "Data" folder. If the Errors Only option is selected, an entry will appear only for files that incurred some type of error during the extraction (discussed below). Regardless of the Error Logging Mode selection, a summary line will always be displayed at the end of the batch.

To extract/process a batch of daily files, first make certain that the daily files are in the correct location (typically c: IDART Files\Data). When processing daily files for a lane for the first time, a good practice is to always validate the daily files. Select the Validate Only option, the preferred Error Logging Mode, and click the Process button. Once validated, switch the Processing Mode to Extract, and click the Process button again. Processing the daily files may take some time, depending on the number of files. Processed daily files will automatically be moved from the "Data" resource folder to the "Processed" resource folder, unless errors were encountered.

Once completed, refresh the Site Metrics Pane by clicking on the country, and then on the site in the Navigation Pane. The values in the Site Metrics Pane should now be updated to reflect

- Earliest Date, the earliest file date existing in the SQL database;

- Latest Date, the latest file date existing in the SQL database;

- Data Files, the number of daily files processed;

- Oversize, the number of daily files that were larger than $8 \mathrm{MB}$;

- Empty, the number of daily files that were smaller than $5 \mathrm{kB}$;

- Bad Format, the number of daily files that had unexpected formats; and

- Missing, the number of daily files missing between the Earliest and Latest File Dates.

Ideally, there will be no Oversized, Empty, Bad Format, or Missing daily files. DART will flag oversized, empty, and bad format daily files as errors in the Results Pane and automatically move them to the corresponding resource folder (e.g. c:IDART Files\Oversize). Figure 11 shows the Site Metric Pane after processing three daily files. The summary message is visible in the Results Pane. Once daily files are processed, the Analysis Tools may be used. 


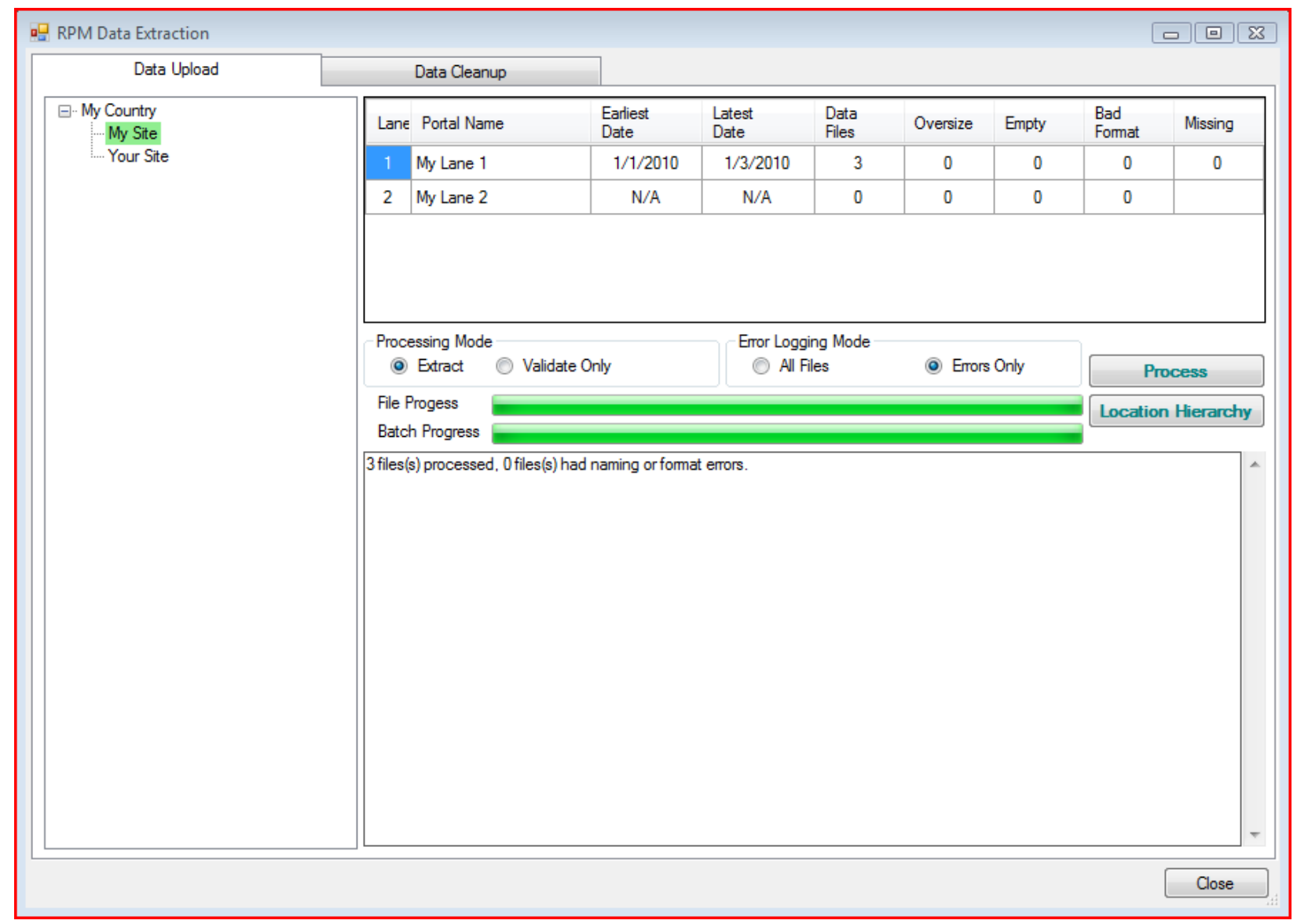

Fig. 11. RPM Extraction Example

\section{Data Cleanup}

Only a limited amount of data can be stored in the data repository when using SQL Express. Currently, the limit for SQL Express 2008 is $10 \mathrm{~Gb}$. To avoid overfilling the database, data should be periodically deleted from DART's data repository. This is accomplished in the Data Cleanup tab near the top of the RPM Data Extraction window.

The Data Cleanup tab is similar to the Data Upload tab. The country, site, and lane appear at the left in the Navigation Pane; the Site Metrics Pane, again at the top; and the Results Pane, at the bottom. Instead of Extraction Options being in the center of the page, however, there are three radio buttons that specify date intervals, two date selection boxes (the second visible only when needed), a graphical representation of the amount and dates of data that will be deleted, and a Delete button (Fig. 12). 


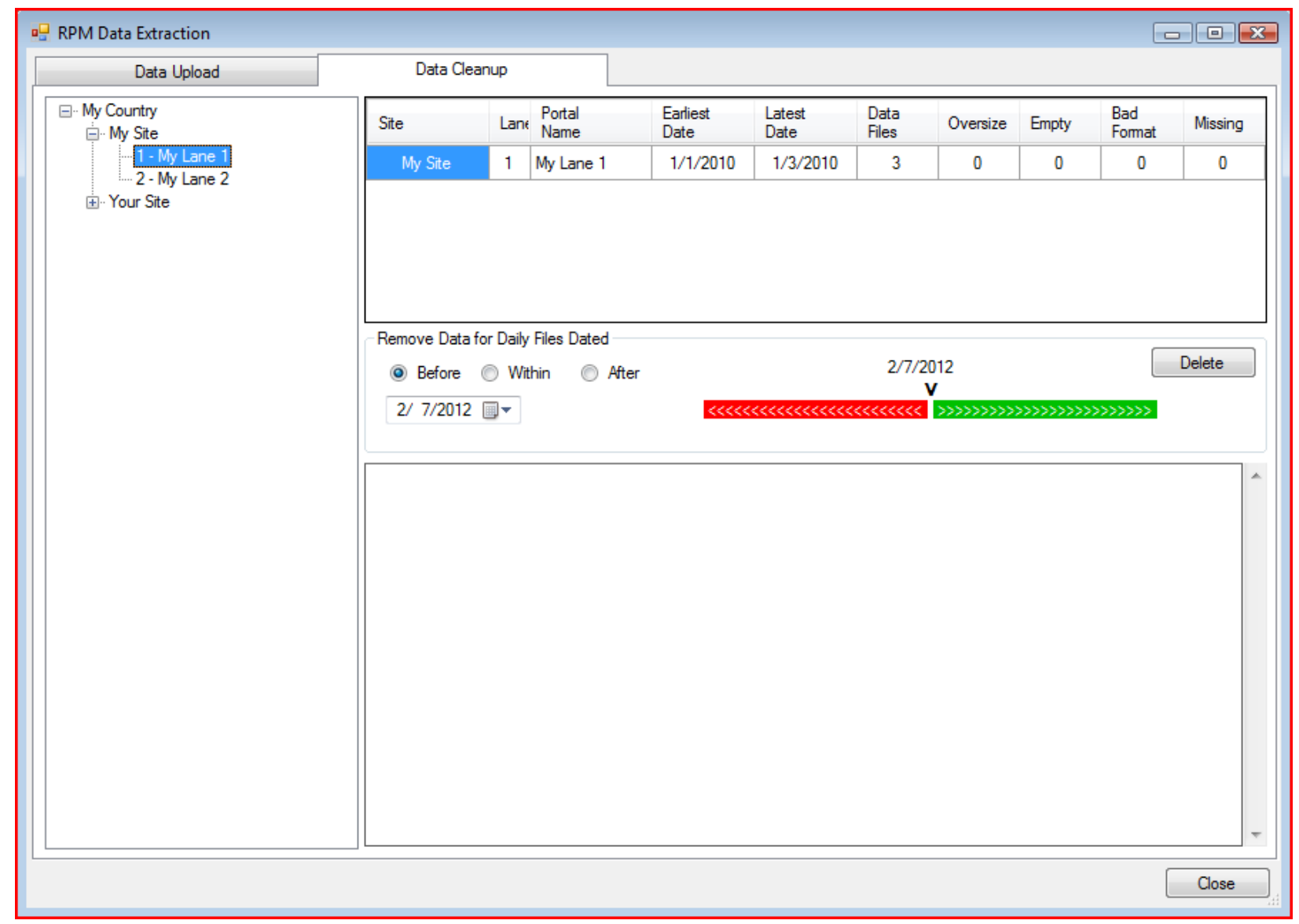

Fig. 12. RPM Data Extraction - Data Cleanup

The amount of data deleted depends on two factors: the hierarchy level highlighted in the Navigation Pane, and the dates of the data to be deleted. Data can be deleted at the lane, site, or country level depending on what is highlighted in the Navigation Pane. For example, data related to a single lane can be deleted by highlighting the lane in the Navigation Pane. Likewise, data related to an entire site or country can be deleted by highlighting that site or country, respectively, in the Navigation Pane. Note: If data is deleted for a selected item, the data for anything subordinate to it will also be deleted (e.g., deleting a site will delete the data for all lanes associated with it).

The amount of data deleted also depends on the dates selected with the radio buttons, as shown in Fig. 13. The graphical representation of the data indicates the specific data to be deleted (in red) based on the selection criteria.

- Selecting Before will delete data for dates prior to, but not including, the selected date

- Selecting Within will delete data for dates between, and including, the selected dates

- Selecting After will delete data for dates after, but not including, the selected date 


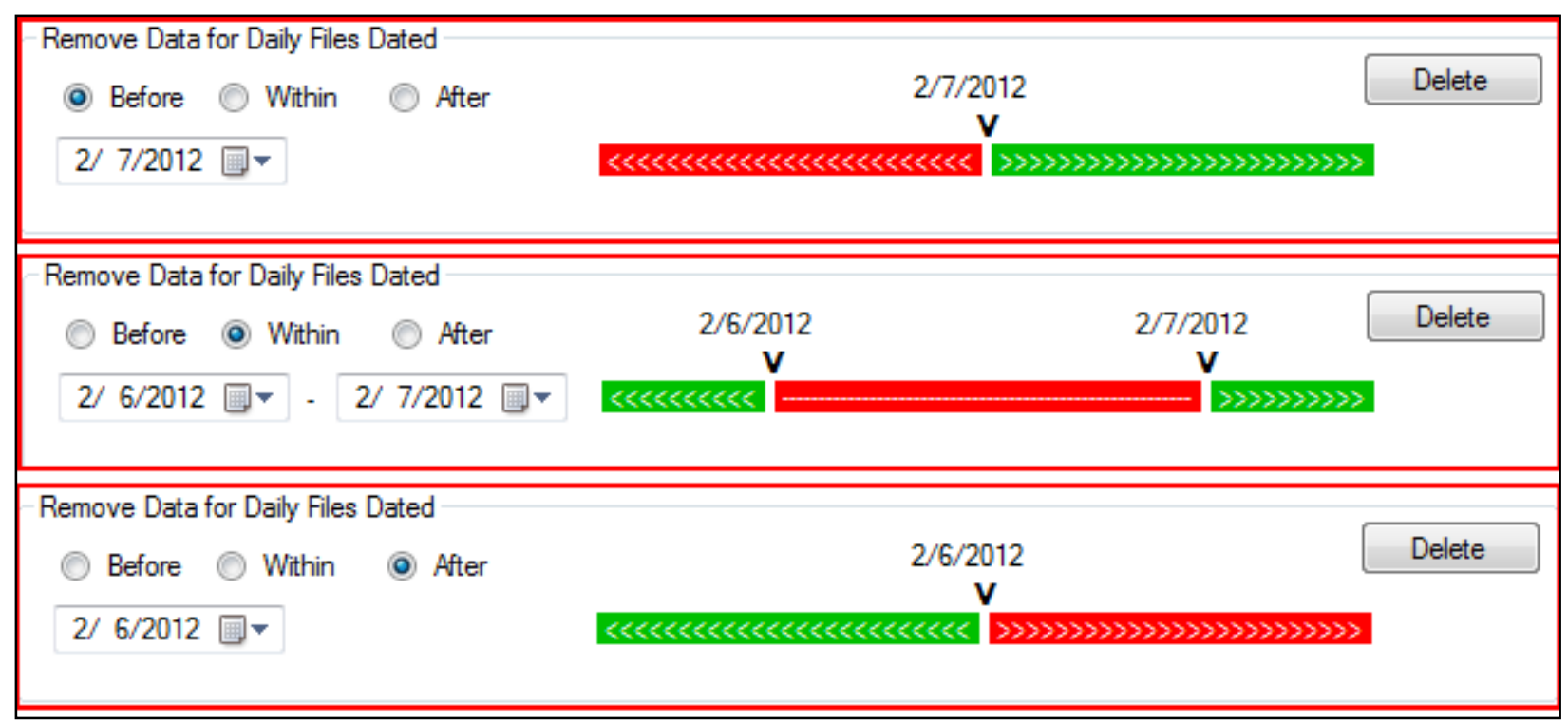

Fig. 13. Examples of Data Cleanup Date Range

To delete data, first choose the level from which the data should be deleted, and select it in the Navigation Pane on the left of the Data Cleanup window. Then select a date range (it is most common to delete data older than a particular date, by selecting the Before option). Choose a date. When you are ready to delete the data, click the Delete button. The message shown in Fig. 14 is presented as a safety measure.

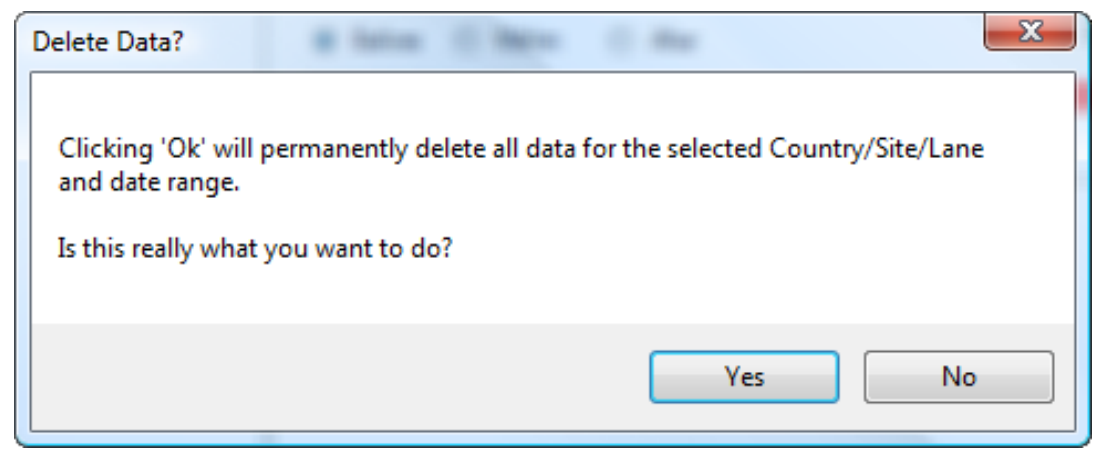

Fig. 14. Warning Prior to Data Deletion

Select Yes to proceed with the data deletion, or select No to select some other combination. Clicking Yes will permanently delete the data, update the message area, and redisplay the data for the selected country. If at any time you want to look at this data again, the daily files must be reprocessed in the Data Upload window.

\subsection{Analysis Tools}

Analysis Tools contains options for examining the data extracted from the daily files. These tools provide the user with useful metrics and plots and allow the user to manipulate the data to extract useful operational and functional information. Two analysis tools are available: the lane snapshot and the data dashboard. 


\subsubsection{Lane Snapshot}

The Lane Snapshot provides a one-page functional summary of a single lane. In most cases, the state of health of a single RPM can be determined through evaluation of the Lane Snapshot. The Lane Snapshot, shown on the following page in Fig. 15, is divided into four main sections: Navigation Pane on the left, Lane Metrics at the top of the screen, Plots in the center of the screen, and Settings at the bottom of the screen. Each of these sections is described in the following paragraphs. To view the snapshot for a specific lane, first choose the date range of interest in the area at the top of the Lane Snapshot screen. Selecting the date range before selecting the lane in the Navigation Pane increases the speed at which DART displays the correct data.

After the date range is set, expand the country and site in the Navigation Pane. Click on a lane to display the snapshot.

\section{Navigation Pane}

The Navigation Pane is used to select a specific lane for analysis. All metrics, plots, and settings shown in the Lane Snapshot are specific to the lane selected in the Navigation Pane.

\section{Lane Metrics}

The Lane Metrics portion of the Lane Snapshot contains a number of tables that display data associated with the daily files. Five sections comprise the Lane Metrics: Data Availability, Occupancy Metrics, Gamma Metrics, Neutron Metrics, and Fault Metrics. In addition to providing metrics and settings, DART highlights unexpected or undesirable values for specific metrics. The parameters susceptible to highlighting, and the criteria, are provided here.

\section{Data Availability}

The Data Availability section (Fig. 16) contains information specifically about the daily files (dates of, presence of, validity of, etc.). Table 1 lists the Data Availability metrics and describes each one. If a value for oversized, empty, bad format, or missing files is nonzero, that value will be highlighted to draw attention to the issue.

\begin{tabular}{|lc|}
\hline Data Availability & \\
Earliest Date & $10 / 1 / 2011$ \\
Latest Date & $10 / 31 / 2011$ \\
Total Files & 31 \\
Oversize Files & 0 \\
Empty Files & 0 \\
Bad Format Files & 0 \\
Missing Files & 0 \\
\hline
\end{tabular}

Fig. 16. Lane Snapshot - Data Availability 


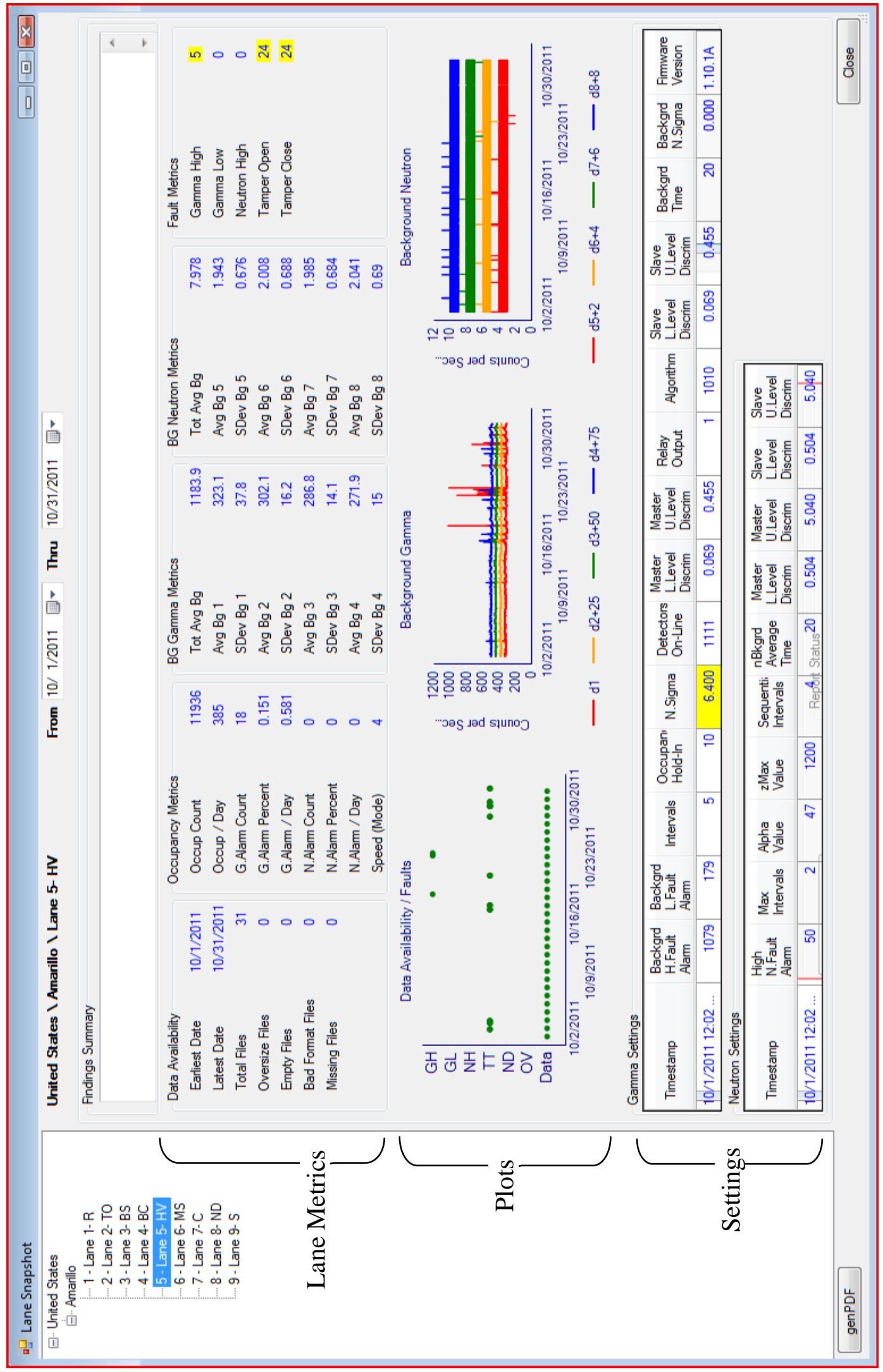

Fig. 15. Lane Snapshot 
Table 1. Lane Snapshot - Data Availability Metrics

\begin{tabular}{|l|l|}
\hline Metric & \multicolumn{1}{|c|}{ Description } \\
\hline Earliest Date & Date of first daily file available within date range selected \\
\hline Latest Date & Date of last daily file available within date range selected \\
\hline Total Files & Total number of daily files within date range selected \\
\hline Oversized Files & Total number of oversized $(>8 \mathrm{MB})$ daily files within date range selected \\
\hline Empty Files & Total number of empty $(<5 \mathrm{kB})$ daily files within date range selected \\
\hline Bad Format Files & Total number of bad format (unprocessed) daily files within date range selected \\
\hline Missing Files & Total number of missing daily files within date range selected \\
\hline
\end{tabular}

\section{Occupancy Metrics}

The Occupancy Metrics table (Fig. 17) lists details related to the occupancies experienced by the lane. For example, the occupancy count (Occup Count) gives the total number of occupancies seen by the RPM for the date range selected, while the Gamma Alarm Count (G. Alarm Count) gives the total number of alarms seen by the RPM for the date range selected. A complete list of these metrics with definitions is given in Table 2. The highlighted criteria, where applicable, are included in the table.

\begin{tabular}{|ll|}
\hline Occupancy Metrics & \\
Occup Count & 11936 \\
Occup / Day & 385 \\
G.Alarm Count & 18 \\
G.Alarm Percent & 0.151 \\
G.Alarm / Day & 0.581 \\
N.Alarm Count & 0 \\
N.Alarm Percent & 0 \\
N.Alarm / Day & 0 \\
Speed (Mode) & 4 \\
\hline
\end{tabular}

Fig. 17. Lane Snapshot Occupancy Metrics

Table 2. Lane Snapshot - Occupancy Metrics

\begin{tabular}{|l|l|}
\hline Occupancy Metric & \multicolumn{1}{|c|}{ Definition } \\
\hline Occup Count & Total number of occupancies during date range selected \\
\hline Occup / Day & Total number of occupancies divided by number of days in date range \\
\hline G. Alarm Count & Total number of gamma alarms during date range selected \\
\hline G. Alarm Percent & $\begin{array}{l}\text { Gamma alarm count divided by occupancy count as percent; gamma alarm rates } \\
\text { greater than 3\% will be highlighted in the Lane Snapshot }\end{array}$ \\
\hline G. Alarm / Day & Total number of gamma alarms divided by number of days in date range \\
\hline N. Alarm Count & $\begin{array}{l}\text { Total number of neutron alarms during date range selected; a neutron count that is } \\
\text { nonzero will be highlighted in the Lane Snapshot }\end{array}$ \\
\hline N. Alarm Percent & $\begin{array}{l}\text { Neutron alarm count divided by occupancy count as percent; a neutron alarm rate } \\
\text { greater than zero will be highlighted in the Lane Snapshot }\end{array}$ \\
\hline N. Alarm / Day & Total number of neutron alarms divided by number of days in date range \\
\hline Speed (Mode) & $\begin{array}{l}\text { Most common speed recorded during date range selected; a mode of 10 or greater } \\
\text { will be highlighted in the Lane Snapshot }\end{array}$ \\
\hline
\end{tabular}




\section{Gamma Metrics}

The Gamma Metrics (BG Gamma Metrics) table (Fig. 18) lists the average gamma background, in counts per second (cps), for each individual detector assembly over the date range selected (Avg Bg X). The standard deviation for each background count rate (Std Dev $\mathrm{X}$ ) is included in this table. In addition to the individual background responses, the total, or sum, of all the detector averages is also given (Tot Avg $\mathrm{Bg}$ ). These values should be fairly consistent across all of the detector assemblies.

Neutron Metrics

\begin{tabular}{|ll|}
\hline BG Neutron Metrics & \\
Tot Avg Bg & 7.978 \\
Avg Bg 5 & 1.943 \\
SDev Bg 5 & 0.676 \\
Avg Bg 6 & 2.008 \\
SDev Bg 6 & 0.688 \\
Avg Bg 7 & 1.985 \\
SDev Bg 7 & 0.684 \\
Avg Bg 8 & 2.041 \\
SDev Bg 8 & 0.69 \\
\hline
\end{tabular}

Fig. 19. Lane Snapshot Neutron Metrics

\begin{tabular}{|ll|}
\hline BG Gamma Metrics & \\
Tot Avg Bg & 1183.9 \\
Avg Bg 1 & 323.1 \\
SDev Bg 1 & 37.8 \\
Avg Bg 2 & 302.1 \\
SDev Bg 2 & 16.2 \\
Avg Bg 3 & 286.8 \\
SDev Bg 3 & 14.1 \\
Avg Bg 4 & 271.9 \\
SDev Bg 4 & 15 \\
\hline
\end{tabular}

Fig. 18. Lane Snapshot Gamma Metrics

The Neutron Metrics (BG Neutron Metrics) table (Fig. 19) lists the average neutron background, in cps, for each individual detector assembly over the date range selected (Avg Bg X). Included in this table is the standard deviation for each background count rate (Std Dev X). In addition to the individual background responses, the total, or sum, of all the detector averages is also given (Tot Avg Bg). Again, these values should be consistent across all of the detector assemblies.

\section{Fault Metrics}

The Fault Metrics table (Fig. 20) shows the number of gamma high (GH), gamma low (GL), neutron high (NH), tamper open (TT), and tamper close (TC) faults experienced by the RPM over the date range selected. Ideally, these will be zero. If any of these values are nonzero, they will be highlighted.

\begin{tabular}{|c|c|}
\hline \multicolumn{2}{|l|}{ Fault Metrics } \\
\hline Gamma High & 5 \\
\hline Gamma Low & 0 \\
\hline Neutron High & 0 \\
\hline Tamper Open & 24 \\
\hline Tamper Close & 24 \\
\hline
\end{tabular}

Fig. 20. Lane Snapshot - Fault Metrics

\section{Plots}

The Plots section of the Lane Snapshot provides a graphical interpretation of the Data Availability, Gamma Metrics, Neutron Metrics, and Fault Metrics tables. 


\section{Data Availability/Fault}

The Data Availability/Faults plot (Fig. 21) shows the date for which daily files were present; the daily files, if any, that were oversized (OV) or empty (ND); and the dates on which each fault condition occurred. The user can zoom in on any region by clicking and dragging the mouse over the region of interest. To exit the zoomed-in view, click the circles at the left and/or top of the graph axes.

\section{Background Gamma}

The Background Gamma plot (Fig. 22) shows the background profiles for each individual detector assembly. The values are offset, to more easily distinguish each detector, in the following manner:

- Detector $1(\mathrm{~d} 1)$ is not offset

- Detector 2 (d2) is offset by 25 counts (25 counts are added to the actual background counts for plotting purposes)

- Detector $3(\mathrm{~d} 3)$ is offset by 50 counts (50 counts are added to the actual background counts for plotting purposes)

- Detector 4 (d4) is offset by 75 counts (75 counts are added to the actual background counts for plotting purposes)

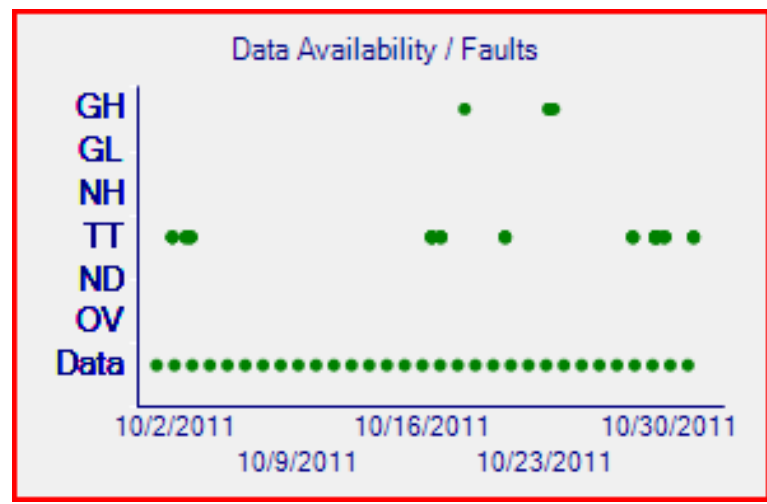

Fig. 21. Lane Snapshot - Data Availability/Faults Plot

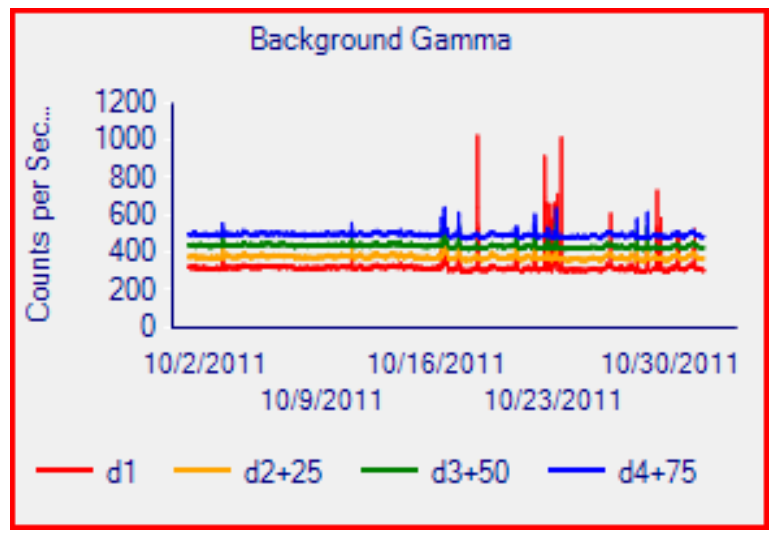

Fig. 22. Lane Snapshot - Background Gamma

The user can zoom in on a region of this plot by clicking and dragging the mouse over the region of interest. To exit the zoomed-in view, click the circles at the left and/or top of the graph axes. 


\section{Background Neutron}

The Background Neutron plot (Fig. 23) shows the background profiles for each individual detector assembly. The values are offset, to more easily distinguish each detector, in the following manner:

- Detector 5 (d5) is offset by 2 counts (2 counts are added to the actual background counts for plotting purposes)

- Detector 6 (d6) is offset by 4 counts (4 counts are added to the actual background counts for plotting purposes)

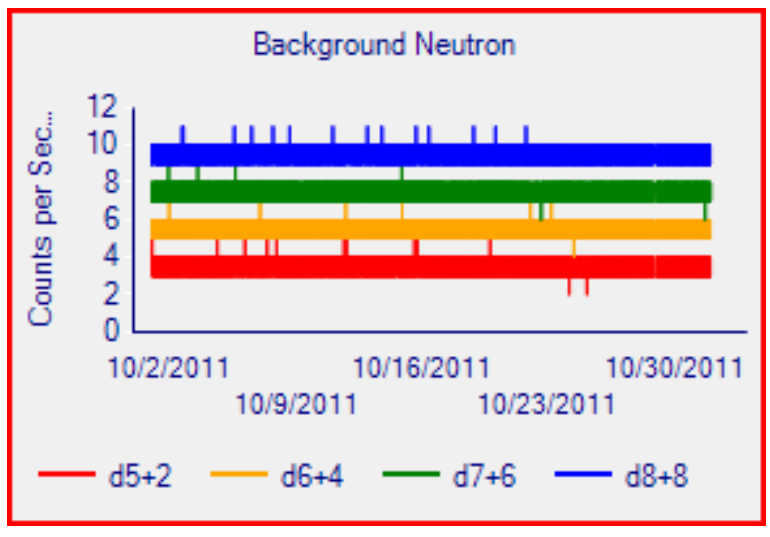

Fig. 23. Lane Snapshot - Background Neutron

- Detector 7 (d7) is offset by 6 counts $(6$ counts are added to the actual background counts for plotting purposes)

- Detector 8 (d8) is offset by 8 counts $(8$ counts are added to the actual background counts for plotting purposes)

The user can zoom in on a region of this plot by clicking and dragging the mouse over the region of interest. To exit the zoomed-in view, click the circles at the left and/or top of the graph axes.

\section{Settings}

The Settings portion of the Lane Snapshot is composed of two tables: Gamma Settings and Neutron Settings. The settings are displayed as shown in Fig. 24 with the gamma settings listed first and the neutron settings listed second. The gamma settings and their descriptions can be found in Table 3, while the neutron settings and their descriptions can be found in Table 4 .

\begin{tabular}{|c|c|c|c|c|c|c|c|c|c|c|c|c|c|c|c|}
\hline \multicolumn{16}{|l|}{ Gamma Settings } \\
\hline Timestamp & $\begin{array}{l}\text { Backgrd } \\
\text { H.Fault } \\
\text { Alarm }\end{array}$ & $\begin{array}{l}\text { Backgrd } \\
\text { L.Fault } \\
\text { Alam }\end{array}$ & Intervals & $\begin{array}{l}\text { Occupanc } \\
\text { Hold-In }\end{array}$ & N.Sigma & $\begin{array}{l}\text { Detectors } \\
\text { On-Line }\end{array}$ & $\begin{array}{l}\text { Master } \\
\text { L.Level } \\
\text { Discrim }\end{array}$ & $\begin{array}{l}\text { Master } \\
\text { U.Level } \\
\text { Discrim }\end{array}$ & $\begin{array}{l}\text { Relay } \\
\text { Output }\end{array}$ & Algorithm & $\begin{array}{l}\text { Slave } \\
\text { L.Level } \\
\text { Discrim }\end{array}$ & $\begin{array}{l}\text { Slave } \\
\text { U.Level } \\
\text { Discrim }\end{array}$ & $\begin{array}{l}\text { Backgrd } \\
\text { Time }\end{array}$ & $\begin{array}{l}\text { Backgrd } \\
\text { N.Sigma }\end{array}$ & $\begin{array}{l}\text { Firmware } \\
\text { Version }\end{array}$ \\
\hline 10/1/2011 12:02 AM & 1079 & 179 & 5 & 10 & 6.400 & 1111 & 0.069 & 0.455 & 1 & 1010 & 0.069 & 0.455 & 20 & 0.000 & $1.10 .1 \mathrm{~A}$ \\
\hline \multicolumn{16}{|l|}{ Neutron Settings } \\
\hline Timestamp & $\begin{array}{l}\text { High } \\
\text { N.Fault } \\
\text { Alarm }\end{array}$ & $\begin{array}{l}\text { Max } \\
\text { Intervals }\end{array}$ & $\begin{array}{l}\text { Alpha } \\
\text { Value }\end{array}$ & $\begin{array}{l}\text { zMax } \\
\text { Value }\end{array}$ & $\begin{array}{l}\text { Sequentia } \\
\text { Intervals }\end{array}$ & $\begin{array}{l}\mathrm{nBkg} \text { Brd } \\
\text { Average } \\
\text { Time }\end{array}$ & $\begin{array}{l}\text { Master } \\
\text { L.Level } \\
\text { Discrim }\end{array}$ & $\begin{array}{l}\text { Master } \\
\text { U.Level } \\
\text { Discrim }\end{array}$ & $\begin{array}{l}\text { Slave } \\
\text { L.Level } \\
\text { Discrim }\end{array}$ & $\begin{array}{l}\text { Slave } \\
\text { U.Level } \\
\text { Discrim }\end{array}$ & & & & & \\
\hline 10/1/2011 12:02 AM & 50 & 2 & 47 & 1200 & 4 & 20 & 0.504 & 5.040 & 0.504 & 5.040 & & & & & \\
\hline
\end{tabular}

Fig. 24. Lane Snapshot - Settings 
Table 3. Lane Snapshot - Gamma Settings

\begin{tabular}{|l|l|}
\hline \multicolumn{1}{|c|}{ Setting } & \multicolumn{1}{c|}{ Description } \\
\hline Timestamp & Date and time of entry in daily file \\
\hline $\begin{array}{l}\text { Background H Fault } \\
\text { Alarm }\end{array}$ & $\begin{array}{l}\text { Threshold at which monitor will enter Gamma High (GH) fault condition (based } \\
\text { on a single detector) }\end{array}$ \\
\hline $\begin{array}{l}\text { Background L Fault } \\
\text { Alarm }\end{array}$ & $\begin{array}{l}\text { Threshold at which monitor will enter Gamma Low (GL) fault condition (based } \\
\text { on a single detector) }\end{array}$ \\
\hline Intervals & Number of 200 ms intervals to "look back" after start of an occupancy \\
\hline Occupancy Hold-In & $\begin{array}{l}\text { Number of } 200 \mathrm{~ms} \text { intervals monitor will maintain occupancy after end of } \\
\text { occupancy }\end{array}$ \\
\hline Nsigma & Alarm threshold \\
\hline Detectors On-line & 1 indicates detector is online, 0 indicates detector is offline \\
\hline Master LLD & Master lower level discriminator - cuts out signal at lower energy range \\
\hline Master ULD & Master upper level discriminator - cuts out signal at upper energy range \\
\hline Relay Output & 1 indicates local alarms are on (lights and horns), 0 indicates alarms are off \\
\hline Algorithm & $\begin{array}{l}\text { Dictates which detectors or combination of detectors contribute to alarm } \\
\text { decisions }\end{array}$ \\
\hline Slave LLD & Slave lower level discriminator - cuts out signal at lower energy range \\
\hline Slave ULD & Slave upper level discriminator - cuts out signal at upper energy range \\
\hline Background Time & Background counting time for the system \\
\hline Background Nsigma & Threshold for “throw-through" alarms \\
\hline Firmware Version & Indicates which firmware version is loaded in RPM \\
\hline
\end{tabular}

Table 4. Lane Snapshot - Neutron Settings

\begin{tabular}{|l|l|}
\hline \multicolumn{1}{|c|}{ Setting } & \multicolumn{1}{c|}{ Description } \\
\hline Timestamp & Date and time of entry in daily file \\
\hline $\begin{array}{l}\text { High N Fault } \\
\text { Alarm }\end{array}$ & $\begin{array}{l}\text { Threshold at which monitor will enter Gamma High (GH) fault condition (based on a } \\
\text { single detector) }\end{array}$ \\
\hline $\begin{array}{l}\text { Maximum } \\
\text { Intervals }\end{array}$ & Maximum number of one second intervals for alarm decision \\
\hline Alpha Value & Value used to select alarm and background values, used to express false alarm rate \\
\hline zMax Value & Alarm threshold used after MAX intervals is reached with no alarm decision \\
\hline $\begin{array}{l}\text { Sequential } \\
\text { Intervals }\end{array}$ & $\begin{array}{l}\text { Number of consecutive 200 ms intervals above background needed to alarm or enter } \\
\text { neutron high (NH) fault condition }\end{array}$ \\
\hline $\begin{array}{l}\text { nBackground } \\
\text { Average Time }\end{array}$ & Counting time for establishing neutron background \\
\hline Master LLD & Master lower level discriminator - cuts out signal at lower energy range \\
\hline Master ULD & Master upper level discriminator - cuts out signal at upper energy range \\
\hline Slave LLD & Slave lower level discriminator - cuts out signal at lower energy range \\
\hline Slave ULD & Slave upper level discriminator - cuts out signal at upper energy range \\
\hline
\end{tabular}


If the settings found in the daily files are consistent with default (widely accepted) values that are hardwired into the DART software, the setting appears in white. If, however, the setting differs from an expected value, it is highlighted in yellow. This is meant to draw attention to a potentially incorrect setting. The potentially highlighted settings are the following:

\section{Gamma Settings}

- Upper level discriminators

- Lower level discriminators

- Intervals

- Occupancy Hold-in

- Detectors On-line

- Algorithm

- Background Time

- Background nSigma

- Firmware Version Suffix

\section{Neutron Settings}

- Upper level discriminators

- Lower level discriminators

- zMax

In the event that the settings have changed over the date range selected, a scroll bar will appear on the right side of the settings table. Click to the bottom of the list for the most current settings.

Again, the highlighting mechanism in the Lane Snapshot is an important feature. It gives the user the ability to quickly look at the information related to a single lane and determine whether or not further analysis is required.

\subsubsection{Data Dashboard}

The Data Dashboard is the more advanced option for detailed data analysis. The information provided in the Lane Snapshot is also available, albeit not on one single page, in the Data Dashboard. In addition to the metrics/plots available in the Lane Snapshot, the Data Dashboard also allows the user to review more detailed background profiles, individual alarm profiles with Poisson probabilities, traffic flow through the lane, and various other data. These analysis functions are discussed in this section.

To navigate to the Data Dashboard, click the Data Dashboard option from the DART Main Menu. The Data Dashboard is divided into three main sections: Lane Metrics at the top of the screen, Charting Options in the middle of the screen, and Settings at the bottom of the screen. To view data in the Data Dashboard, a country, site, and lane must be selected from the Criteria Selection drop-down menus at the top of the screen. Additionally, the date range must be selected. Once the criteria are set, click the exclamation mark button to the right to retrieve the data. A screenshot of the Data Dashboard is shown in Fig. 25. 


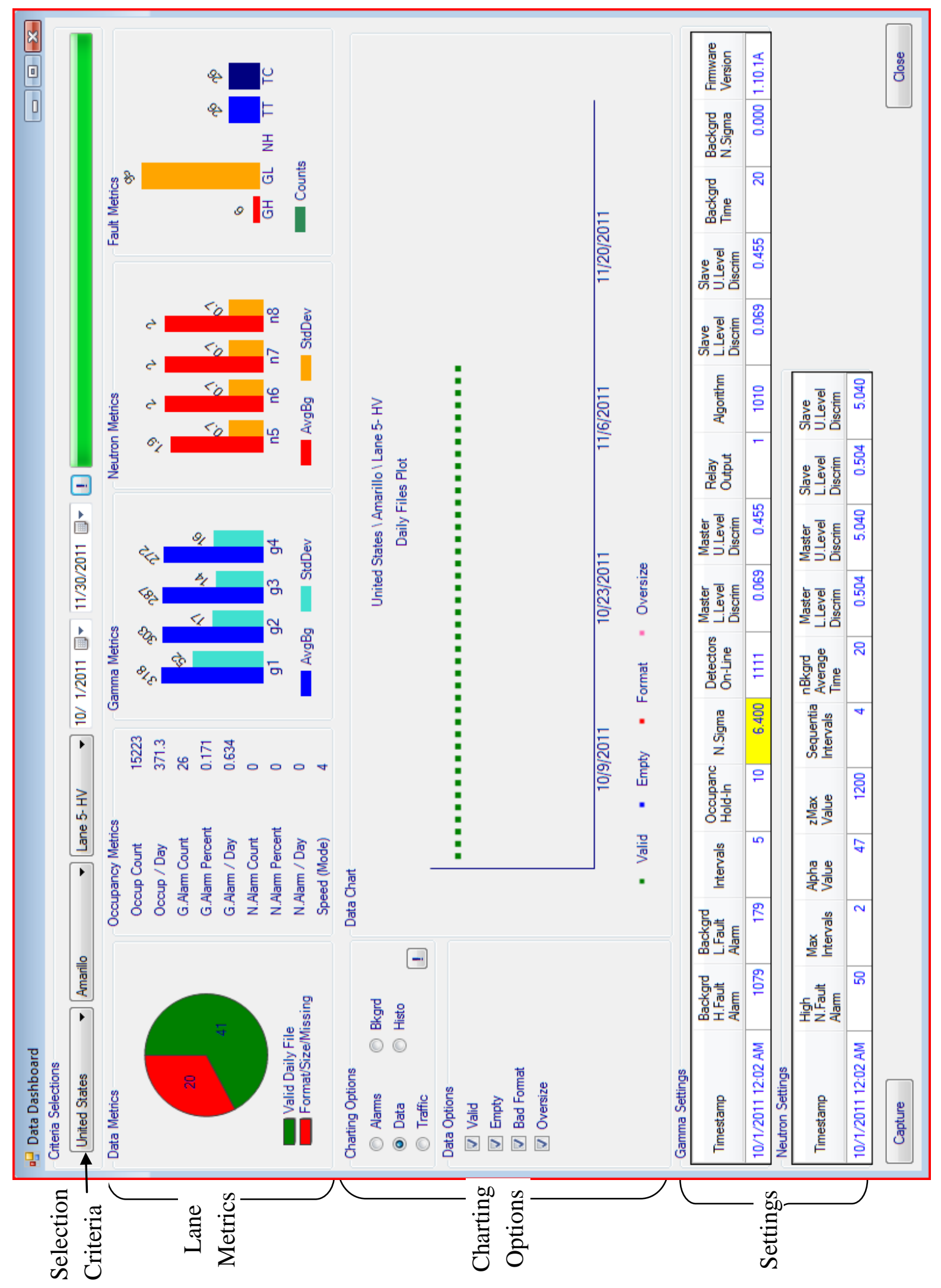

Fig. 25. Data Dashboard 


\section{Lane Metrics}

The Lane Metrics portion of the Data Dashboard encompasses a number of graphical plots and tables showing data associated with the daily files. Five sections comprise the Lane Metrics: Data Metrics, Occupancy Metrics, Gamma Metrics, Neutron Metrics, and Fault Metrics.

\section{Data Metrics}

The Data Metrics section (Fig. 26) contains a pie chart that displays the number of valid daily files (in green) and the number of missing or corrupt (oversized, empty, bad format) daily files (in red) processed during the date range selected.

\section{Occupancy Metrics}

\begin{tabular}{|ll|}
\hline Occupancy Metrics & \\
Occup Count & 11936 \\
Occup / Day & 385 \\
G.Alarm Count & 18 \\
G.Alarm Percent & 0.151 \\
G.Alarm / Day & 0.581 \\
N.Alarm Count & 0 \\
N.Alarm Percent & 0 \\
N.Alarm / Day & 0 \\
Speed (Mode) & 4 \\
\hline
\end{tabular}

Fig. 27. Data Dashboard Occupancy Metrics
The Occupancy Metrics table (Fig. 27) lists details related to the occupancies experienced by the lane. For example, the occupancy count (Occup Count) is the total number of

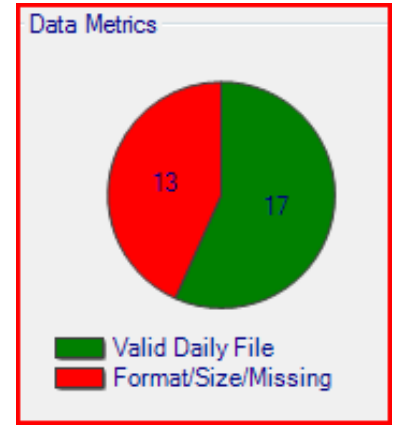

Fig. 26. Data Dashboard Data Metrics occupancies seen by the RPM for the date range selected, while the Gamma Alarm Count (G. Alarm Count) is the total number of alarms seen by the RPM for the date range selected. A complete list of these metrics with definitions is given in Table 5.

Table 5. Data Dashboard - Occupancy Metrics

\begin{tabular}{|l|l|}
\hline Occupancy Metric & \multicolumn{1}{|c|}{ Definition } \\
\hline Occup Count & Total number of occupancies during date range selected \\
\hline Occup / Day & Total number of occupancies divided by number of days in date range \\
\hline G. Alarm Count & Total number of gamma alarms during date range selected \\
\hline G. Alarm Percent & Gamma alarm count divided by occupancy count as percent \\
\hline G. Alarm / Day & Total number of gamma alarms divided by number of days in date range \\
\hline N. Alarm Count & Total number of neutron alarms during date range selected \\
\hline N. Alarm Percent & Neutron alarm count divided by occupancy count as percent \\
\hline N. Alarm / Day & Total number of neutron alarms divided by number of days in date range \\
\hline Speed (Mode) & Most common speed recorded during date range selected \\
\hline
\end{tabular}

Note that the mode of the speed (e.g., the most commonly recorded speed) is reported, rather than the average. ORNL's experience in data analysis has shown the mode speed to be more relevant than the average speed, which can be artificially thrown off by just a few artificially high speed readings of $100 \mathrm{mph}$. 


\section{Gamma Metrics}

The Gamma Metrics chart (Fig. 28) is a bar graph showing the average gamma background, in cps, for each individual detector assembly over the date range selected. These bars are dark blue. The light blue bars represent the standard deviation. These values should be fairly consistent across all the detector assemblies.

\section{Neutron Metrics}

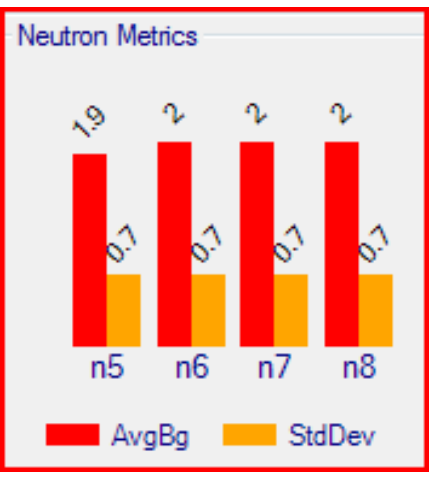

Fig. 29. Data Dashboard Neutron Metrics

\section{Fault Metrics}

The Fault Metrics chart (Fig. 30) shows the number of gamma high $(\mathrm{GH})$, gamma low (GL), neutron high (NH), tamper open (TT), and tamper close (TC) faults experienced by the RPM over the date range selected. Ideally, all will be zero.

\section{Charting Options}

The Neutron Metrics chart (Fig. 29), although similar to the Gamma Metrics chart, displays a graphical interpretation of the average neutron back-

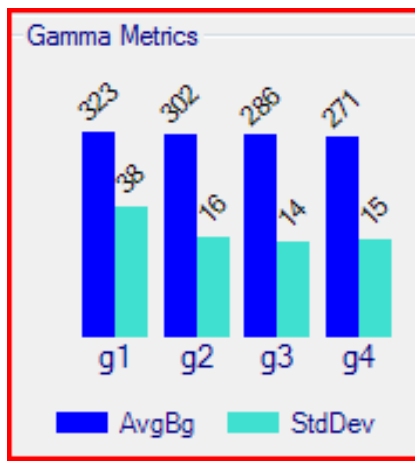

Fig. 28. Data Dashboard Gamma Metrics ground, in cps, for each individual detector assembly over the date range selected. These background rates are shown in red, whereas standard deviation is shown in orange. Again, these values should be consistent across all the detector assemblies.

The Charting Options section of the Data Dashboard is where the bulk of the analysis is performed. Under the Charting Options section, radio buttons indicate the types of information that can be analyzed (i.e., Data, Background Profiles (Bkgrd), Alarms, Histogram Options (Histo), and Traffic). Once a Charting Option is selected, the exclamation mark button must be clicked to update the interface. After updating, additional plotting options may be available. When all options are selected, the data will be plotted in the chart window in the center of the Data Dashboard. These options are explained in detail here.

During the data analysis, any image plotted in the Data Dashboard may be captured/saved by clicking the Capture button located at the bottom of the Data Dashboard. These images can be attached to the Monthly Country Report at a later time. The images will be saved automatically in the "Images" resource folder (typically c:IDART Files Images). 


\section{Data}

The Data option allows the user to obtain information about the daily files. Daily files are represented by colored dots in the Data Chart. If there is not a dot for a specific day, no daily file was received. The default Data Options are Valid, Empty, Bad Format, and Oversize. If these options remain selected, the dots will be color coordinated according to the following criteria:

$$
\begin{aligned}
& \text { Green } \quad \text { - valid/present daily files } \\
& \text { Blue } \quad-\text { empty daily files (less than } 5 \mathrm{kB} \text { in size) } \\
& \text { Red } \quad-\text { daily files with a bad format (not compatible with DART or does not have a valid } \\
& \quad \text { upload file pattern) } \\
& \text { Pink } \quad-\text { oversized daily files (over } 8 \mathrm{MB} \text { in size) }
\end{aligned}
$$

This Charting Option provides users with a simple way to determine if all the daily files were received, if and when daily files were missing, and if and when problems were encountered with any of the daily files. An example of the Data Charting Option is shown in Fig. 31.

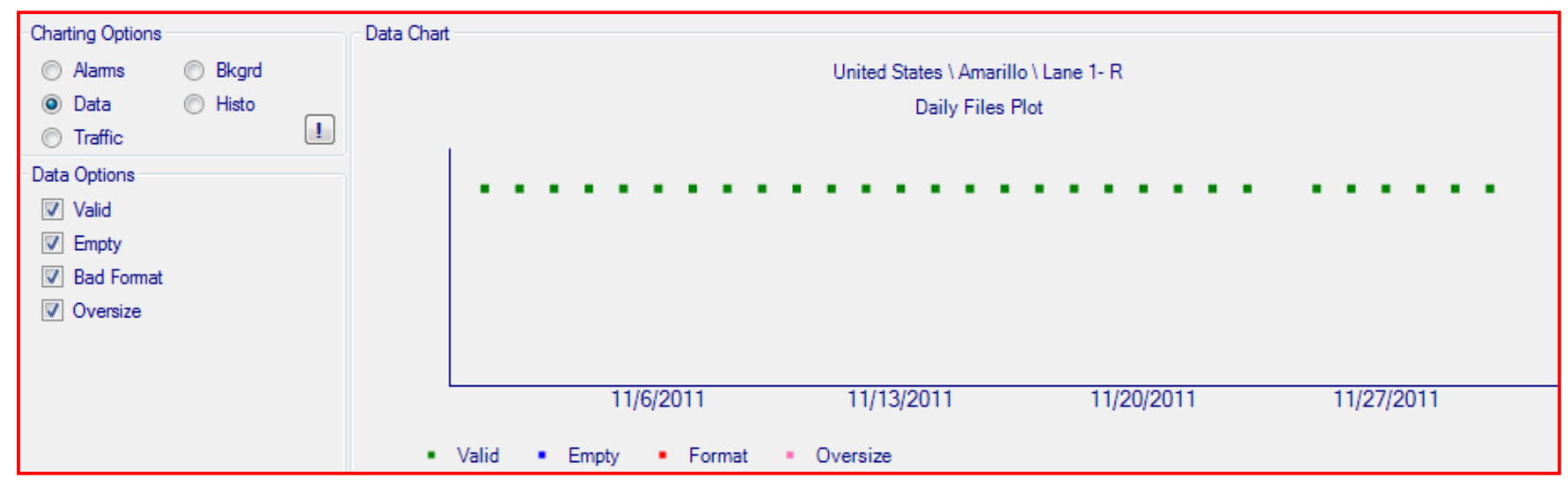

Fig. 31. Charting Options - Data

\section{Background Profiles}

The Background Charting Option allows the user to view background profiles for gamma and/or neutron detector assemblies. The background count rate, that is, the rate when the portal monitor is not occupied, is collected once every 10 minutes; when the monitor is occupied, a rolling sum is used to convert $200 \mathrm{~ms}$ data from the daily file into a 1 second count rate in DART.

Under Background Options, the user has the ability to choose which detectors, individual or in combinations, are displayed in the plot. For example, if the user chooses gamma detectors 1, 2, 3, and 4 (Gam 1, Gam 2, Gam 3, and Gam 4), the individual responses from all four assemblies will be plotted. This is shown in Fig. 32.

Similarly, if neutron detectors 5, 6, 7, and 8, were selected, the plot would show the response from each of the four neutron assemblies. If the two groups were both selected, the gamma and neutron profiles would both be plotted on the same graph. Additionally, pillar sums can be 
plotted (i.e., Gam 1, 2; Gam 3, 4; Neu 5, 6; Neu 7, 8) as well as the sums of all detectors (i.e., Gam Tot and Neu Tot).

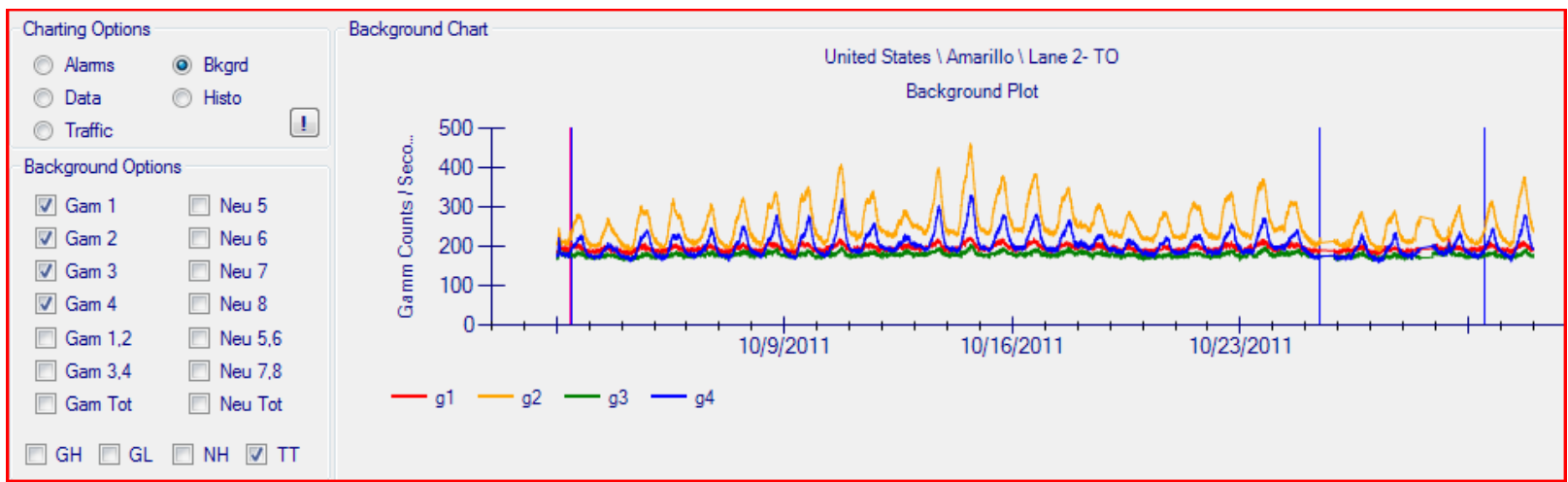

Fig. 32. Charting Options - Background

In addition to plotting the background profiles, DART has the ability to overlay the fault conditions selected under Background Options. In the example above, the tamper fault (TT) box is checked; therefore, blue lines are overlaid on the plot at the time at which they occurred. By pairing the fault condition with the background profile, the user may be able to identify causes of various fault conditions. The user can zoom in on a region of the plot by clicking the mouse and dragging over a portion of the plot to enlarge it. Note that the initial click must be inside the chart area.

\section{Alarm Profiles}

The Alarms Charting Option allows the user to view gamma and neutron alarm profiles. Under Alarm Options RPM, the user can select an alarm for viewing from the drop-down menu as shown in Fig. 33. The alarms are prioritized in the following order: gamma/neutron [GN], neutron $[\mathrm{N}]$, and gamma [G] alarms. The data and time stamp associated with the alarm is also listed in the drop-down menu. Once an alarm is selected, the user must select the appropriate detectors for the plot. This is similar to the Background Charting Option.

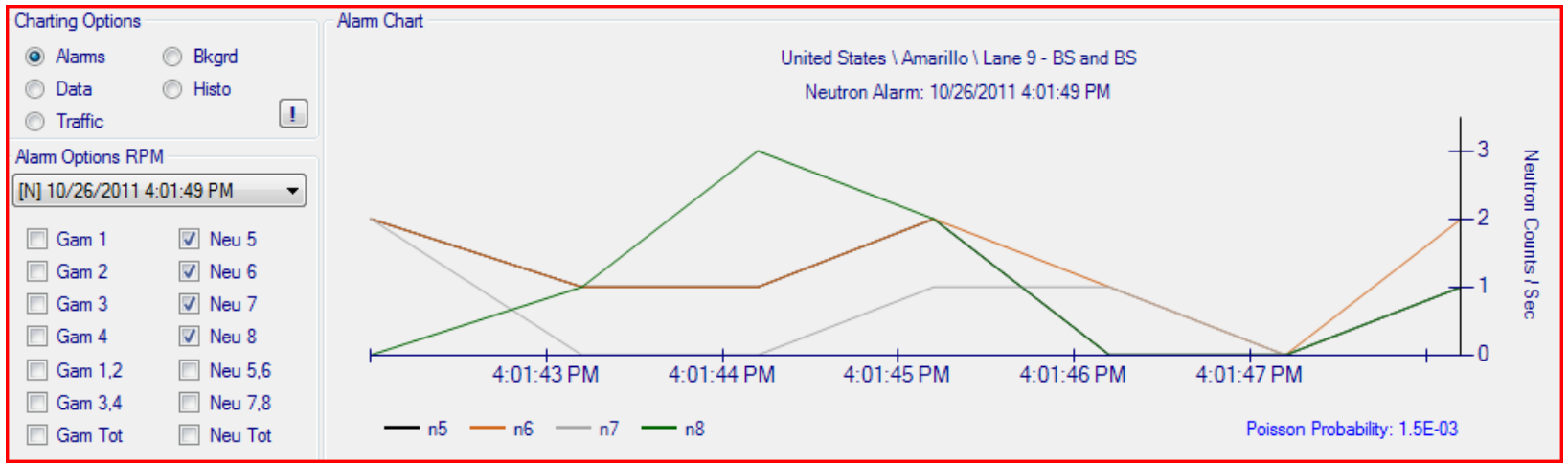

Fig. 33. Charting Options - Alarms 
In addition to the profiles, the Poisson probability is provided in the lower right corner of the plot for neutron alarms. This may help determine whether or not the alarm was statistically significant. Again, the user can zoom in on the plot by dragging the mouse over the region of interest.

\section{Histogram Options}

The Histogram Charting Option allows users to view various distributions of data. The dropdown menu under Histogram Options RPM contains a number of options that reflect the nature of the histogram displayed. The histogram options are background suppression, container length, gamma intervals, and speed. These options are further discussed in the following paragraphs.

In addition to simply selecting the histogram type, the drop-down menu gives access to a table of filter parameters. These parameters allow the user to filter the data included in the histogram. The criteria include histogram ranges and bin sizes. It is important to note that these filters are additive and that the data must meet all the criteria in the table for it to be included in the plot.

Background suppression. Background suppression (Suprs) is the phenomenon that causes the presence of a cargo/container to reduce the background observed by the RPM. The values in the criteria table for low and high are normalized relative to the background values. For example, in Fig. 34, the plot will span from $60 \%$ background suppression to $0 \%$ suppression, which is effectively the average background.

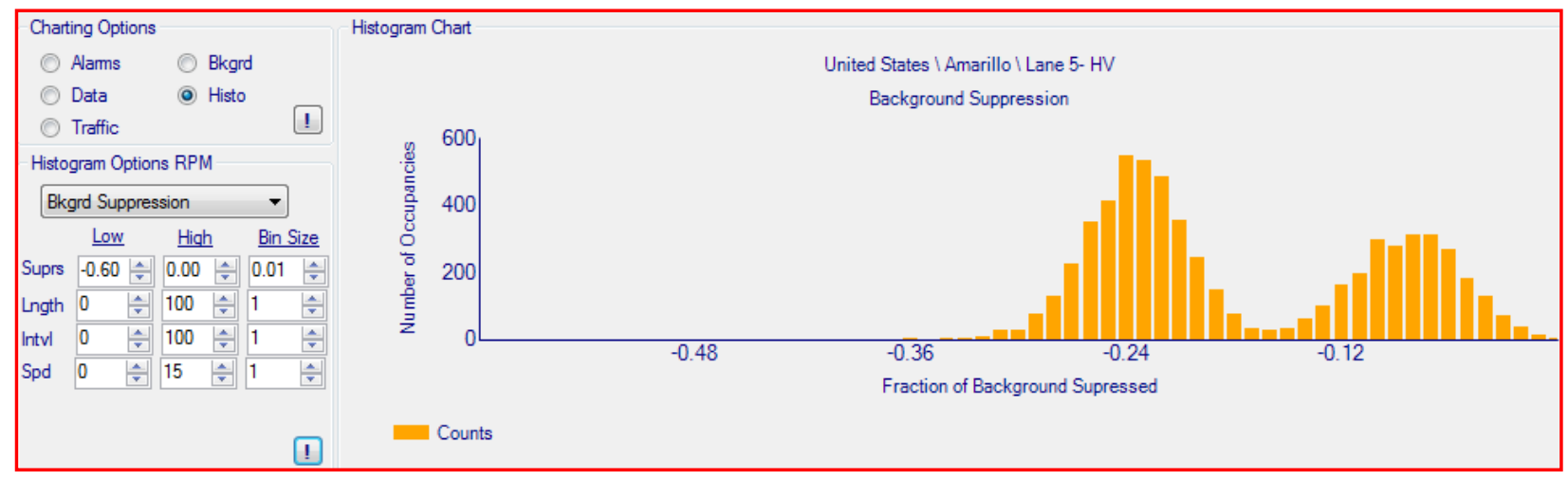

Fig. 34. Charting Options - Histograms - Background Suppression

The example in Fig. 34 shows two averages, one with $\sim 25 \%$ suppression and one with $\sim 10 \%$ suppression. This typically means that two distinct types of cargo are passing through this monitor.

Container length. The container length (Lngth) for the cargo passing through the monitor is calculated with the speed and the number of intervals for each occupancy. The values in the criteria table should be entered in feet. In Fig. 35, the plot shows three averages, one at $\sim 25 \mathrm{ft}$, at $\sim 45 \mathrm{ft}$, and at $\sim 65 \mathrm{ft}$. This is typical of a lane that sees $20 \mathrm{ft}$ containers, $40 \mathrm{ft}$ containers, and long tractor trailers (including a space behind the cab) towing a $40 \mathrm{ft}$ container. 


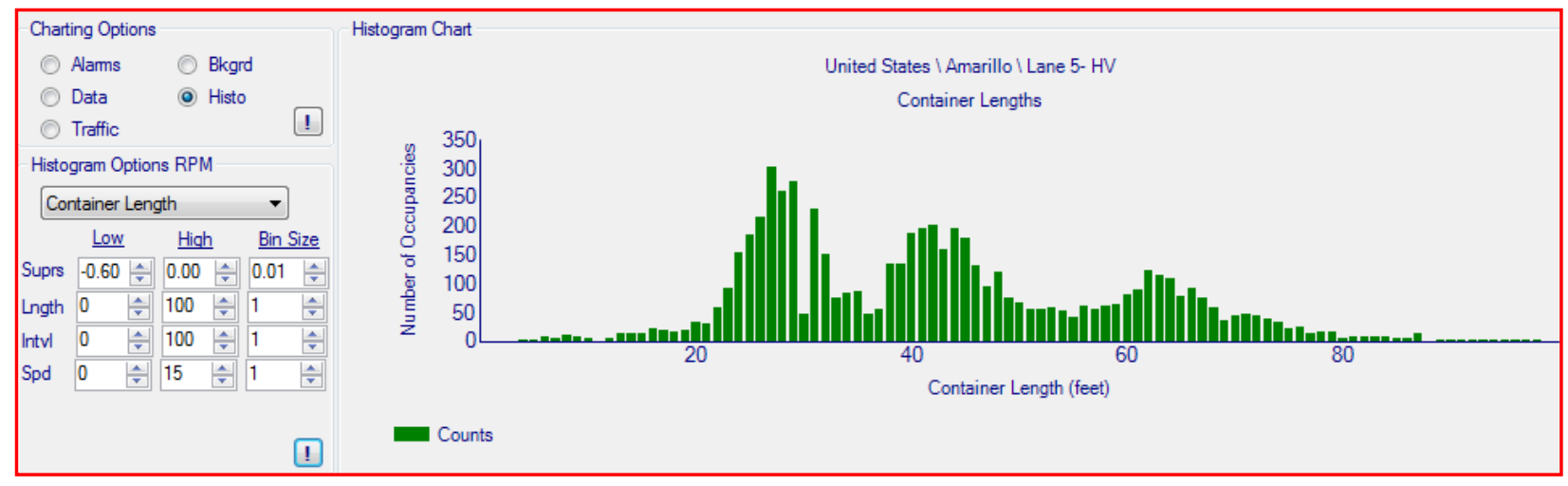

Fig. 35. Charting Options - Histograms - Container Length

Such information may help operators determine the usual types of cargo that pass through each lane, perhaps leading to better traffic flow patterns or operations in general.

Gamma intervals. Gamma intervals (Intvl) refer to the number of $200 \mathrm{~ms}$ intervals recorded for a given occupancy. The values for low and high in the criteria table refer to the number of intervals. In the example shown in Fig. 36, the plot spans from 0 to 100 intervals; any occupancy lasting more than 100 intervals would not be included.

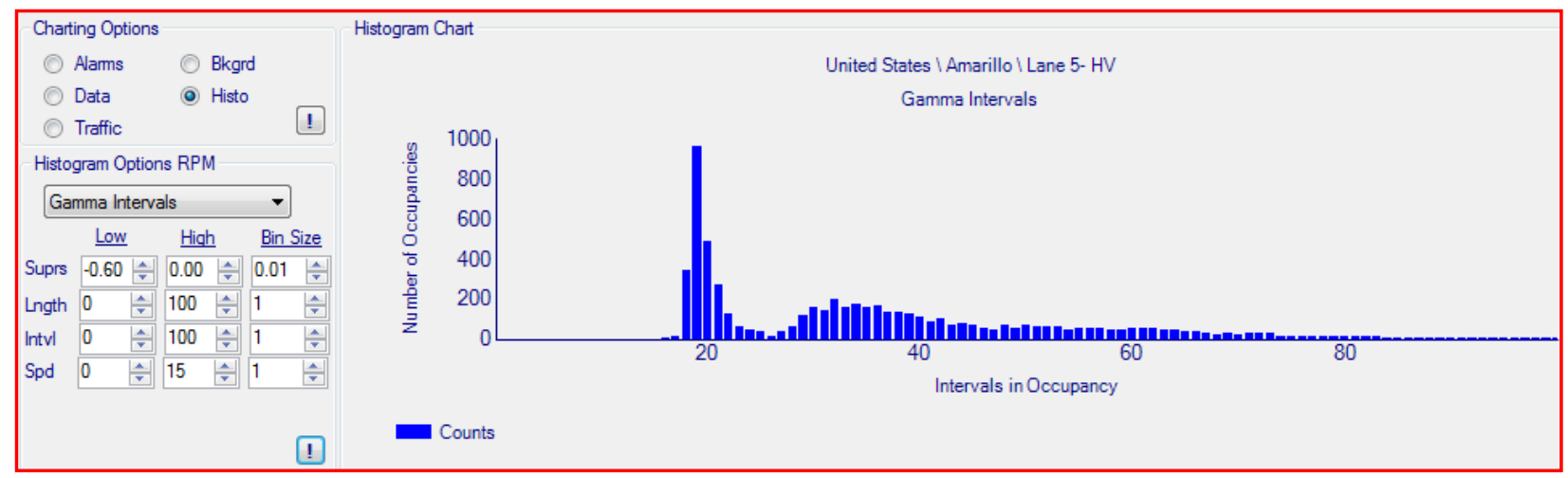

Fig. 36. Charting Options - Histograms - Gamma Intervals

Fig. 36 shows two averages, one at $\sim 20$ intervals and one at $\sim 33$ intervals. Occupancies with fewer than 25 intervals (for vehicle or rail traffic) are typically false occupancies; they indicate an occupancy time of less than 2 seconds. This distribution may help operators understand the rate at which people or cargo pass through the monitors.

Speed. The infrared occupancy sensors provide speed messages for each occupancy. These speed messages are recorded in the daily files and therefore are captured in DART. Occasionally, multiple speed messages are recorded for the same occupancy (due to the cab or windows); however, only the first speed message recorded for an occupancy is used in DART. The values in the criteria table are in miles per hour. In the example shown in Fig. 37, the plot will only include occupancies with recorded speeds between 0 and $15 \mathrm{mph}$. From the plot, it is obvious that most vehicles pass through the monitor at $5 \mathrm{mph}$. This plot can be expanded to see speed messages 
that register speeds upwards of $20 \mathrm{mph}$. Such information may help operators determine whether or not they need to implement better speed control.

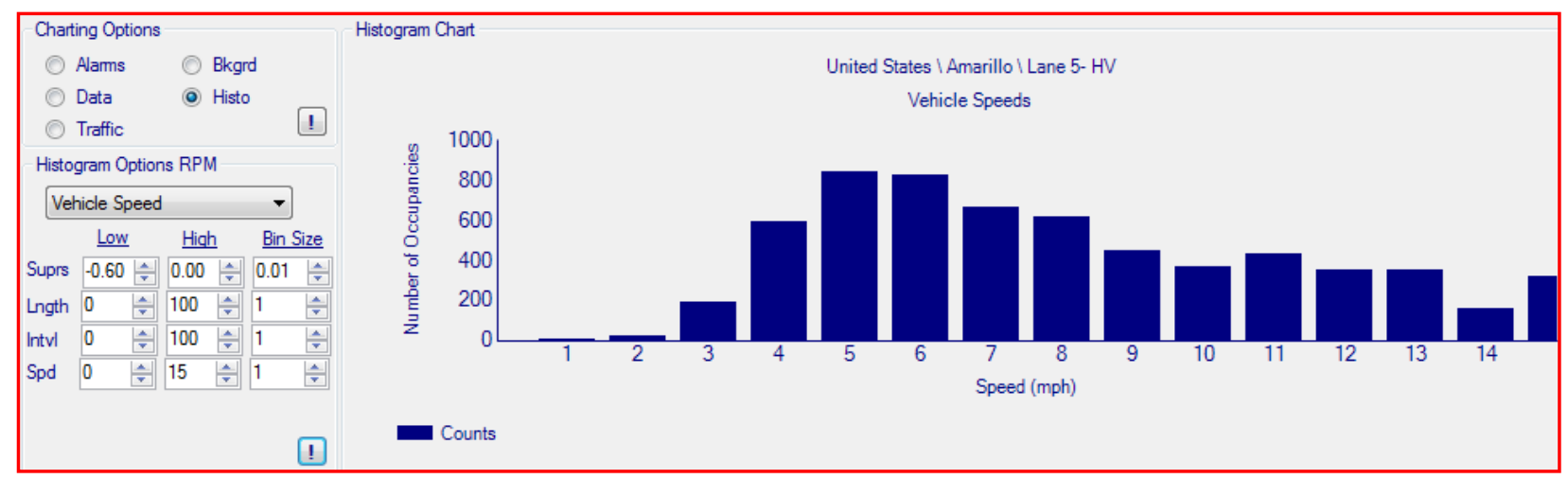

Fig. 37. Charting Options - Histograms - Speed

As demonstrated above, the histograms feature in DART provides advanced analysis capabilities that will assist in making lane operations more efficient. IMPORTANT: Remember that the data included in the plot must meet all the criteria in the options table. As an example, in Fig. 38, the criteria have been changed from default. For the data to be included in this plot, all of the following must have occurred: the cargo caused background suppression (Suprs) between -0.6 and 0 , container length (Lngth) was between 0 and $100 \mathrm{ft}$, occupancy was between 25 and 100 intervals (Intvl), and the cargo travelled at a speed (Spd) between 0 and $15 \mathrm{mph}$.

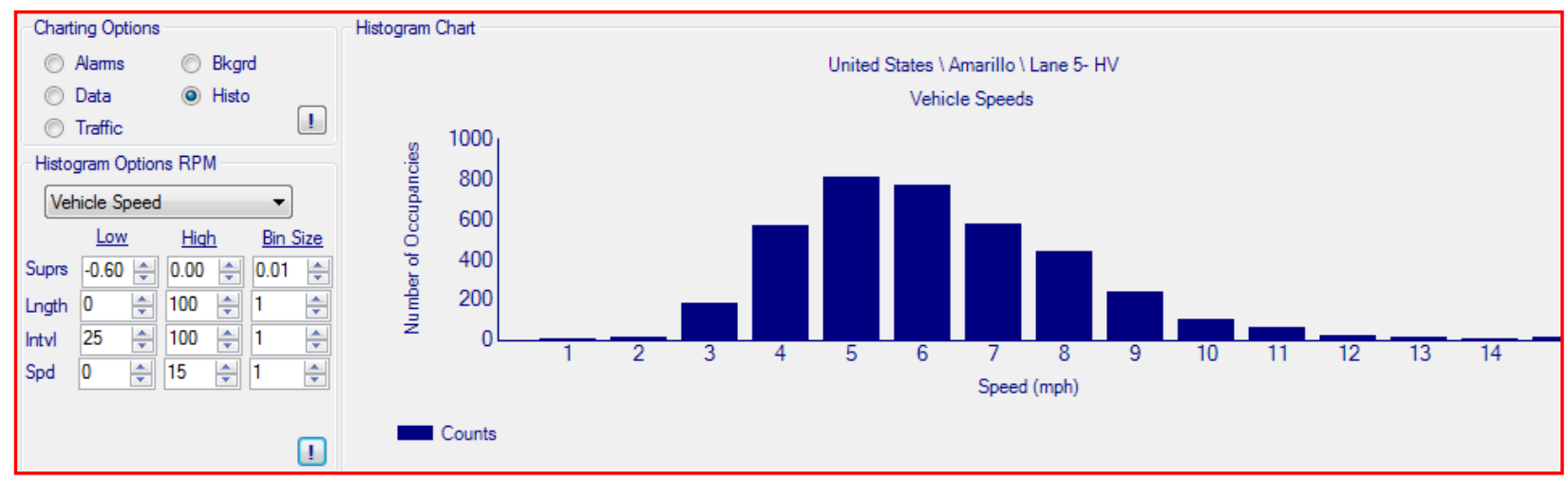

Fig. 38. Charting Options - Histograms

\section{Traffic Distribution}

The Traffic Charting Option allows the user to view the throughput of a lane, in terms of occupancies and alarms. Viewing the throughput of a lane may help operators optimize traffic flow and staffing needs. The Traffic Options, available in the drop-down menu, allow the user to determine how the data will be charted. The options are as follows:

Hour - shows the distribution of occupancies and alarms for every hour. For example, each occupancy that occurred between 6:00 and 7:00, no matter the day, will be combined. This may help operators better understand daily staffing needs. 
Day - shows the distribution of occupancies and alarms for every day in the selected date range. This may help operators understand daily or weekly trends (e.g., traffic flow on weekends).

Month - shows the total number of occupancies and alarms for each month.

Quarter - shows the total number of occupancies and alarms for each quarter.

Yearly - shows the total number of occupancies and alarms for each year.

Note that the date range selected overrides any conflicting Traffic Options selection. For example, choosing "Yearly" will still only show the data within the date range selected. In the bar graph depicting the traffic distribution, the green portion of the bar represents the nonalarming occupancies, while the red portion of the bar represents the alarming occupancies. An example of the throughput by day is given in Fig. 39. Scrolling the mouse over the bar will yield the exact number of occupancies in a pop-up box. The default graph is logarithmic on the $y$-axis, to better show the wide variation between occupancies and alarms.

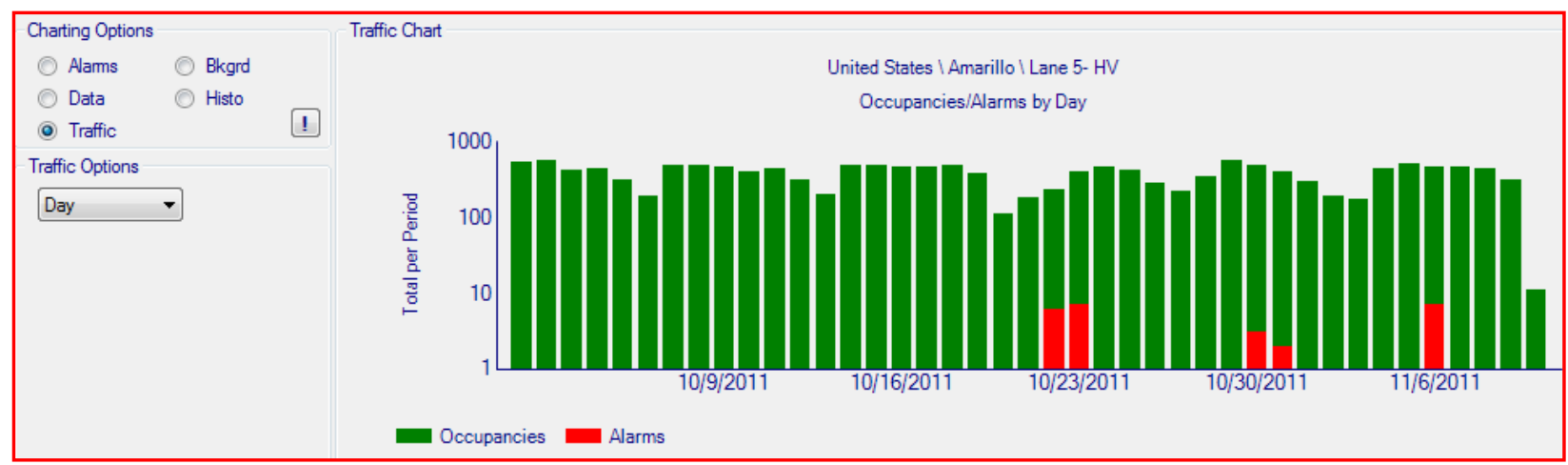

Fig. 39. Charting Options - Traffic

\section{Settings}

The Settings portion of the Data Dashboard is composed of two tables, Gamma Settings and Neutron Settings, and is similar to the Settings table in the Lane Snapshot. The settings are displayed in Fig. 40 with the gamma settings listed first and the neutron settings listed second. The gamma settings and their descriptions can be found in Table 6, while the neutron settings and their descriptions can be found in Table 7.

\begin{tabular}{|c|c|c|c|c|c|c|c|c|c|c|c|c|c|c|c|}
\hline \multicolumn{16}{|l|}{ Gamma Settings } \\
\hline Timestamp & $\begin{array}{l}\text { Backgrd } \\
\text { H.Fault } \\
\text { Alarm }\end{array}$ & $\begin{array}{l}\text { Backgrd } \\
\text { L.Fault } \\
\text { Alarm }\end{array}$ & Intervals & $\begin{array}{l}\text { Occupanc } \\
\text { Hold-ln }\end{array}$ & N.Sigma & $\begin{array}{l}\text { Detectors } \\
\text { On-Line }\end{array}$ & $\begin{array}{l}\text { Master } \\
\text { L.Level } \\
\text { Discrim }\end{array}$ & $\begin{array}{l}\text { Master } \\
\text { U.Level } \\
\text { Discrim }\end{array}$ & $\begin{array}{l}\text { Relay } \\
\text { Output }\end{array}$ & Algorithm & $\begin{array}{l}\text { Slave } \\
\text { L.Level } \\
\text { Discrim }\end{array}$ & $\begin{array}{l}\text { Slave } \\
\text { U.Level } \\
\text { Discrim }\end{array}$ & $\begin{array}{l}\text { Backgrd } \\
\text { Time }\end{array}$ & $\begin{array}{l}\text { Backgrd } \\
\text { N.Sigma }\end{array}$ & $\begin{array}{l}\text { Firmware } \\
\text { Version }\end{array}$ \\
\hline 10/1/2011 12:02 AM & 1079 & 179 & 5 & 10 & 6.400 & 1111 & 0.069 & 0.455 & 1 & 1010 & 0.069 & 0.455 & 20 & 0.000 & $1.10 .1 \mathrm{~A}$ \\
\hline \multicolumn{16}{|l|}{ Neutron Settings } \\
\hline Timestamp & $\begin{array}{l}\text { High } \\
\text { N.Fault } \\
\text { Alarm }\end{array}$ & $\begin{array}{l}\text { Max } \\
\text { Intervals }\end{array}$ & $\begin{array}{l}\text { Alpha } \\
\text { Value }\end{array}$ & $\begin{array}{l}\text { zMax } \\
\text { Value }\end{array}$ & $\begin{array}{l}\text { Sequentia } \\
\text { Intervals }\end{array}$ & $\begin{array}{l}\text { nBkgrd } \\
\text { Average } \\
\text { Time }\end{array}$ & $\begin{array}{l}\text { Master } \\
\text { L.Level } \\
\text { Discrim }\end{array}$ & $\begin{array}{l}\text { Master } \\
\text { U.Level } \\
\text { Discrim }\end{array}$ & $\begin{array}{l}\text { Slave } \\
\text { L.Level } \\
\text { Discrim }\end{array}$ & $\begin{array}{l}\text { Slave } \\
\text { U.Level } \\
\text { Discrim }\end{array}$ & & & & & \\
\hline 10/1/2011 12:02 AM & 50 & 2 & 47 & 1200 & 4 & 20 & 0.504 & 5.040 & 0.504 & 5.040 & & & & & \\
\hline
\end{tabular}

Fig. 40. Data Dashboard - Settings 
Table 6. Data Dashboard - Gamma Settings

\begin{tabular}{|l|l|}
\hline \multicolumn{1}{|c|}{ Setting } & \multicolumn{1}{c|}{ Description } \\
\hline Timestamp & Date and time of entry in daily file \\
\hline $\begin{array}{l}\text { Background H Fault } \\
\text { Alarm }\end{array}$ & $\begin{array}{l}\text { Threshold at which monitor will enter Gamma High (GH) fault condition (based } \\
\text { on a single detector) }\end{array}$ \\
\hline $\begin{array}{l}\text { Background L Fault } \\
\text { Alarm }\end{array}$ & $\begin{array}{l}\text { Threshold at which monitor will enter Gamma Low (GL) fault condition (based } \\
\text { on a single detector) }\end{array}$ \\
\hline Intervals & Number of 200 ms intervals to "look back" after start of an occupancy \\
\hline Occupancy Hold-In & $\begin{array}{l}\text { Number of } 200 \text { ms intervals monitor will maintain occupancy after end of } \\
\text { occupancy }\end{array}$ \\
\hline Nsigma & Alarm threshold \\
\hline Detectors On-line & 1 indicates detector is online, 0 indicates detector is offline \\
\hline Master LLD & Master lower level discriminator - cuts out signal at lower energy range \\
\hline Master ULD & Master upper level discriminator - cuts out signal at upper energy range \\
\hline Relay Output & 1 indicates local alarms are on (lights and horns), 0 indicates alarms are off \\
\hline Algorithm & Dictates which detectors or combination of detectors contribute to alarm decisions \\
\hline Slave LLD & Slave lower level discriminator - cuts out signal at lower energy range \\
\hline Slave ULD & Slave upper level discriminator - cuts out signal at upper energy range \\
\hline Background Time & Counting time for establishing gamma background \\
\hline Background Nsigma & Threshold for “throw-through" alarms \\
\hline Firmware Version & Indicates which firmware version is loaded in RPM \\
\hline
\end{tabular}

Table 7. Data Dashboard - Neutron Settings

\begin{tabular}{|l|l|}
\hline \multicolumn{1}{|c|}{ Setting } & \multicolumn{1}{c|}{ Description } \\
\hline Timestamp & Date and time of entry in daily file \\
\hline High N Fault Alarm & $\begin{array}{l}\text { Threshold at which monitor will enter Gamma High (GH) fault condition (based } \\
\text { on a single detector) }\end{array}$ \\
\hline Maximum Intervals & Maximum number of one second intervals for alarm decision \\
\hline Alpha Value & Value used to select alarm and background values, used to express false alarm rate \\
\hline zMax Value & Alarm threshold used after MAX intervals is reached with no alarm decision \\
\hline Sequential Intervals & $\begin{array}{l}\text { Number of consecutive 200 ms intervals above background needed to alarm or } \\
\text { enter neutron high (NH) fault condition }\end{array}$ \\
\hline $\begin{array}{l}\text { nBackground } \\
\text { Average Time }\end{array}$ & \begin{tabular}{l} 
Counting time for establishing neutron background \\
\hline Master LLD
\end{tabular} Master lower level discriminator - cuts out signal at lower energy range \\
\hline Master ULD & Master upper level discriminator - cuts out signal at upper energy range \\
\hline Slave LLD & Slave lower level discriminator - cuts out signal at lower energy range \\
\hline Slave ULD & Slave upper level discriminator - cuts out signal at upper energy range \\
\hline
\end{tabular}


If the settings found in the daily files are consistent with default (widely accepted) values that are hardwired into the DART software, the setting appears in white. If, however, the setting differs from an expected value, it is highlighted in yellow. This is meant to draw attention to the potentially incorrect setting. The potentially highlighted settings are the following:

\section{Gamma Settings}

- Upper level discriminators

- Lower level discriminators

- Intervals

- Occupancy Hold-in

- Detectors On-line

- Algorithm

- Background Time

- Background nSigma

- Firmware Version Suffix

\section{Neutron Settings}

- Upper level discriminators

- Lower level discriminators

- zMax

In the event that the settings have changed over the date range selected, a scroll bar will appear on the right side of the settings table. Click to the bottom of the list for the most current settings.

The Data Dashboard provides advanced analysis capabilities not available in the Lane Snapshot. By utilizing a combination of these two analysis functions, users have the ability to assess, diagnose, and troubleshoot RPM issues based on the data provided in the daily files.

\subsection{Report Management}

The Report Management function provides an ability to produce structured reports. Currently, the Monthly Country Report is the only reporting feature available outside ORNL.

\subsubsection{Monthly Country Report}

The Monthly Country Report (MCR) is a summary report outlining the important parameters, RPM behaviors, and states of health of each monitor within a specified country. Findings are recorded and summarized in multiple layers. To create a new MCR, click the Monthly Country Report option in the DART Main Menu. The Country Report List will appear (Fig. 41). Choose a country, date range, analyst, and click the New Report button. 


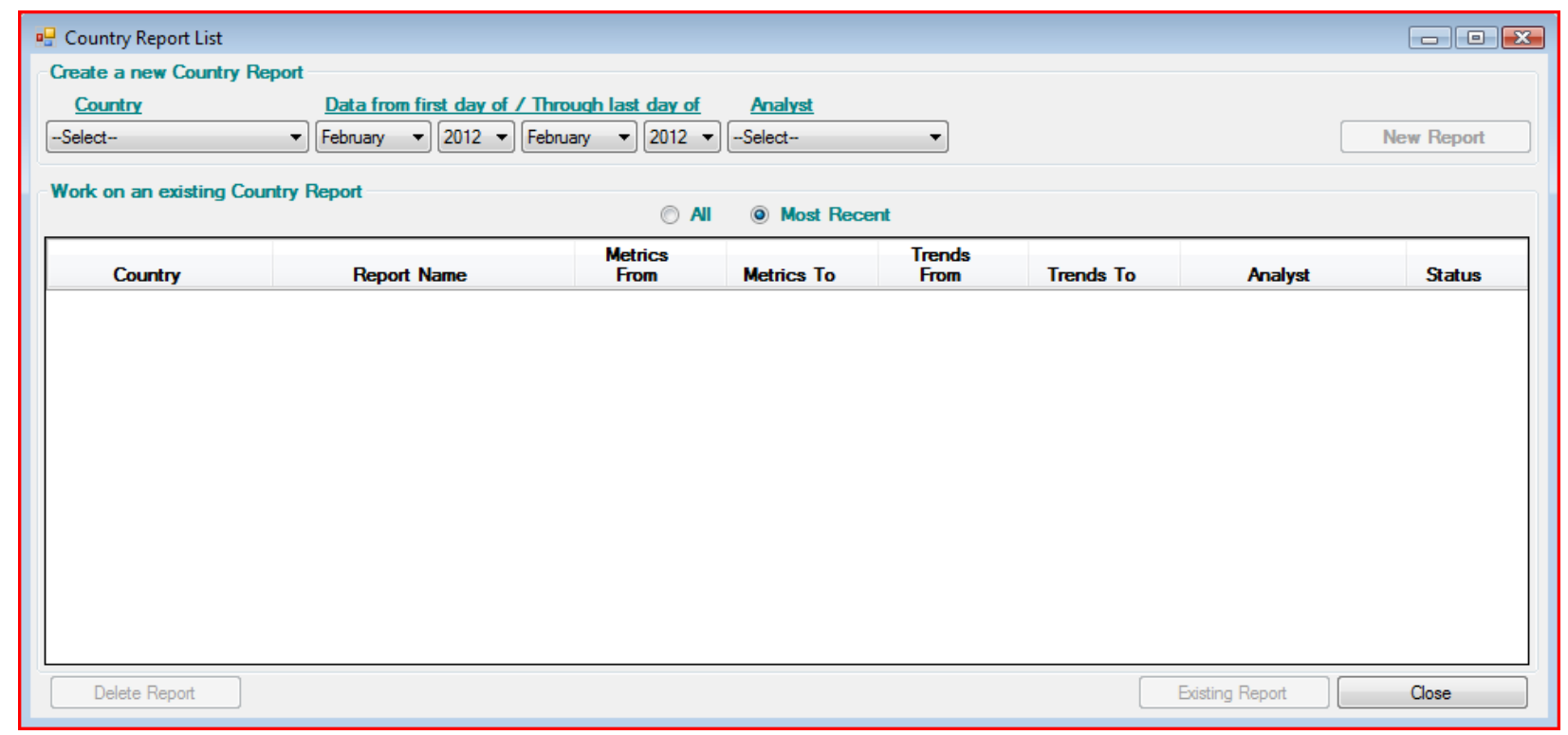

Fig. 41. Monthly country Report - Country Report List

The MCR Report window will open. The Navigation Pane appears on the left, while the analysis window appears on the right. In the Navigation Pane are an Overview section, a list of sites (each having a [+] sign if lanes are contained within that site), and a Summary. Inactive sites (as set in the Location Hierarchy) will not appear in the MCR.

\section{Overview}

The Overview option in the Navigation Pane allows the user to summarize all comments made at the site level into the Overview Comments. By clicking the Roll Up button at the bottom of the window, DART rolls all the comments made in the Site Comments window into the Overview Comments window, breaking the comments into sections based on the site name. It is common to complete the Overview section after the lanes and sites have been reviewed and comments have been added.

\section{Site}

The Site option in the Navigation Pane allows the users to summarize all comments made at the lane level into the Site Comments. By clicking on Roll Up button at the bottom of the window, DART rolls all the comments made in the Findings Summary for each lane into the Site Comments window, breaking the comments into sections based on the lane name. It is common to complete the Site section after the lanes have been reviewed and the findings have been documented.

\section{Lanes}

Expanding the $[+]$ at the site level causes the lanes to appear. Lanes with no traffic type or detector type selected in the Location Hierarchy will not appear in the MCR hierarchy. Upon expanding the $[+]$ at the lane level, two options become available: Snapshot and Images. 


\section{Snapshot}

Clicking on the Snapshot option provides the user with the Lane Snapshot for the lane. This snapshot is identical to the Lane Snapshot available in Analysis functions except that the Plots include data from either the date range selected or the past 3 months, whichever is longer. The metrics will reflect only the data for the date range selected.

In the Snapshot, a Findings Summary is at the top of the window. Comments regarding issues, findings, or concerns may be entered into this field. These comments will be saved automatically in DART, and they will also appear in the Lane Overview window.

\section{Images}

In the event that more details are warranted, DART has the ability to include additional images from the Data Dashboard. By clicking the Images option in the Navigation Pane, an Attach Image (s) option becomes available (Fig. 42). DART automatically looks in the "Images" folder (typically c:IDART FilesImages). Images that were captured in the Data Dashboard (as discussed in Section 3.3.2) are available. Click the image(s) to attach them to the report; the Navigation Pane should refresh to reflect the addition of the new image(s).

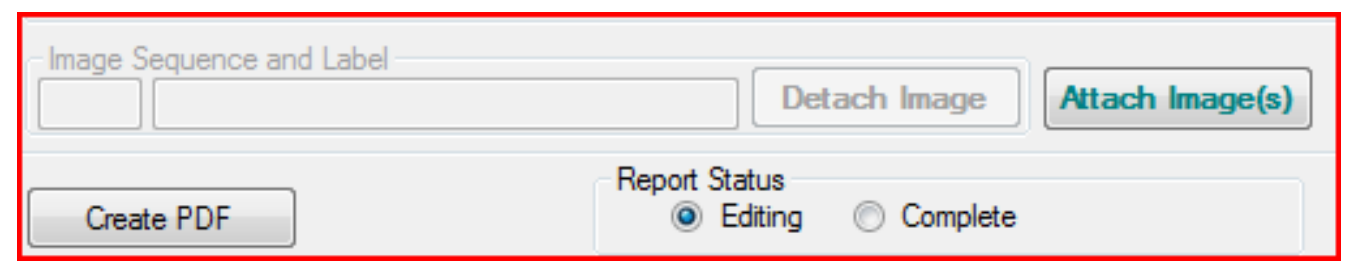

Fig. 42. Monthly Country Report - Images

After the image is attached, its label can be changed to reflect the content of the image. Additional comments about the image can be added to the Image Comments section of the window. Images can also be deleted from the report by clicking on the image in the Navigation Pane, then clicking the Detach Image button.

When the comments for all lanes have been documented, the site comments can be completed. Upon completion of the site comments, the overview comments can be concluded.

\section{Summary}

The Summary table at the end of the Navigation Pane (Table 8) provides a quick summary of key metrics for all the lanes within the country. This table provides a quick and easy method of viewing key metrics for all lanes at one time (e.g., alarm rates). 
Table 8. Monthly Country Report - Summary Table

\begin{tabular}{|l|l|}
\hline Parameter & \multicolumn{1}{|c|}{ Description } \\
\hline Site & Site Name \\
\hline Portal Name & Display Name (from Location Hierarchy) \\
\hline Portal Traffic Type & Intended cargo type (from Lane Attributes) \\
\hline First (in rpt month) & Date of first daily file in report month \\
\hline Last (in rpt month) & Date of last daily file in report month \\
\hline Data Days & Number of daily files present for report month \\
\hline Bad Format & Number of daily files with unexpected format or features \\
\hline G. tot Ave Bkg (cps) & Total average gamma background (sum of all detectors) \\
\hline N. tot Ave Bkg (cps) & Total average neutron background (sum of all detectors) \\
\hline Occup Per Day & Number of occupancies divided by number of days in report month \\
\hline G. Alarm Per Day & Number of gamma alarms divided by number of days in report month \\
\hline N. Alarm Per Day & Number of neutron alarms divided by number of days in report month \\
\hline G. Alarm Percent & Gamma alarm rate for report month \\
\hline N. Alarm Percent & Neutron alarm rate for report month \\
\hline Speed (Mode) & Most common speed registered for all occupancies in report month \\
\hline GH Count & Number of Gamma High faults in report month \\
\hline GL count & Number of Gamma Low faults in report month \\
\hline NH Count & Number of Neutron High faults in report month \\
\hline TT Count & Number of Tamper faults in report month \\
\hline
\end{tabular}

After all comments have been documented in the Monthly Country Report, a PDF can be generated by clicking the Create PDF button at the bottom of the MCR window (see Fig. 42). This may take a few minutes, depending on the number of sites and lanes included in the report. When document generation is complete, click the Complete radio button under Report Status. The MCR will be saved in the "Reports" resource folder (typically c:|DART Files\ Reports (Complete). The user may revisit an existing report in DART by selecting the report and clicking the Existing Report button shown in Fig. 41. 


\section{Appendix A: Troubleshooting Guide for SQL Express Installation}

Users of SQL Express sometimes experience problems when initially connecting to DART. If, after installation, a connection with the database cannot be established, try one or more of the following options:

- Log onto your personal computer as the Administrator. DART creates two files during the initial connection: config.xml and dartData_log.ldf. Occasionally, creating these files requires an elevated level of access

- Ensure that the "instance name" of the SQL Express installation is set to "SQLExpress". This is sometimes an issue for users who have upgraded from a previous version

- Delete "dartData_log.ldf" and try again. This too can be a result of upgrading from a previous version

Users of SQL Express on Windows 7 machines who continue to have connection issues should try the following:

- Locate and delete the "SQLEXPRESS" folder at the end of this path: c: IUsers\[userid]\AppDatalLocallMicrosoftlMicrosoft SQL Server DatalSQLEXPRESS

- Substitute your userid for the bracketed value

- Note that the "AppData" folder is hidden in Windows 7, so it will need to be keyed directly in a browser URL, or into the search path under the start menu

- You may actually want to leave off the "ISQLEXPRESS" so that it comes up in the right window and you can delete it from there

- Try to establish connection in DART again

- Change the Log On used by the SQL Express service

- Start SQLServer Configuration Manager from the start menu

- Select the first entry in the left pane: "SQL Server Services"

- Right click on the first entry in the right pane: "SQL Server (SQLEXPRESS)", pick properties

- If "Log on as:" is set to "Built in account - Network Account", change it to "Local Service", or your user id (under "This Account") as shown in Fig. A.1

- Hit apply, the service will end and restart

- Try again to connect to DART 


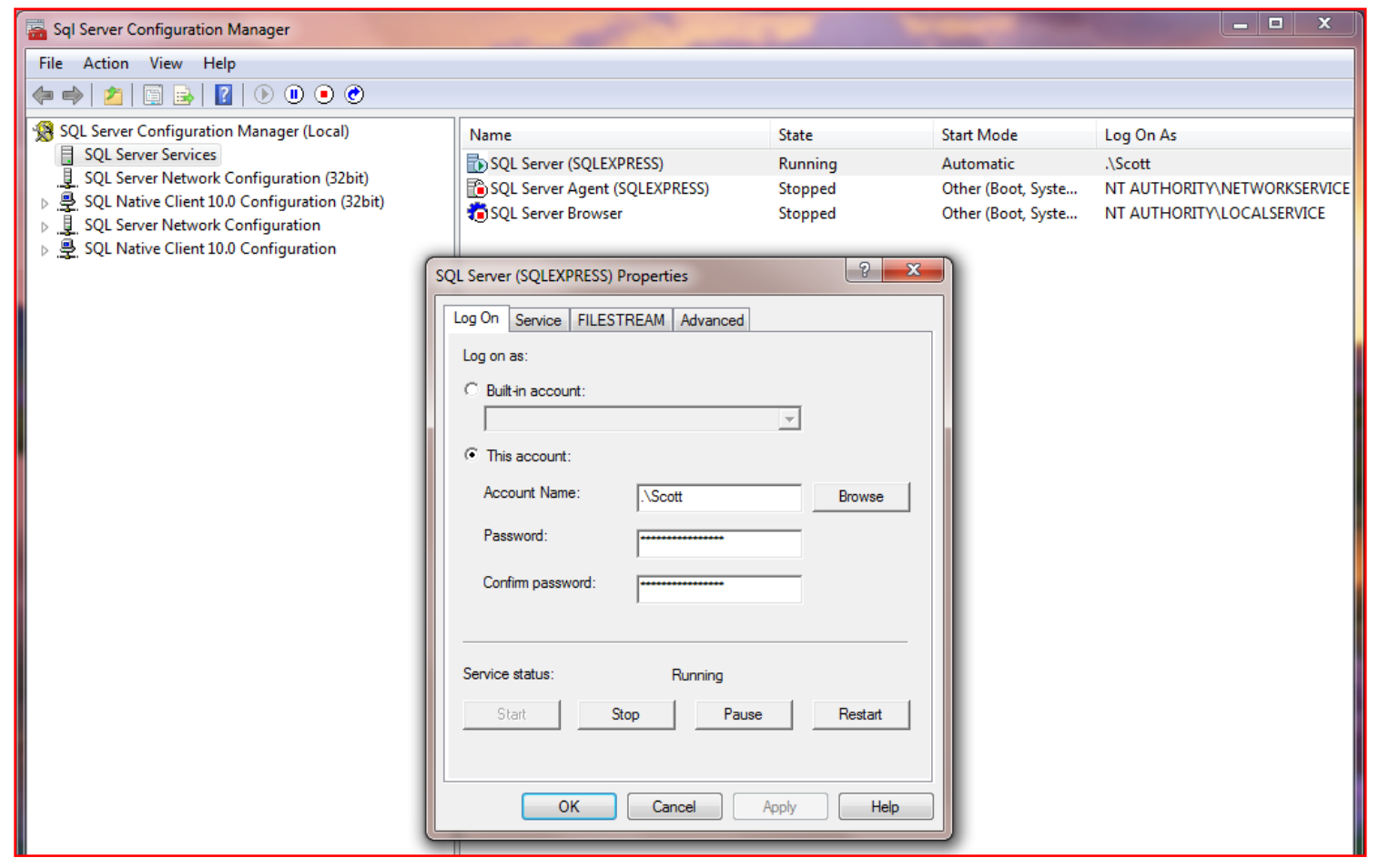

Fig. A.1. SQL Server Management Studio 